\title{
FLOW OF BLUE GLACIER, OLYMPIC MOUNTAINS, WASHINGTON, U.S.A.*
}

\author{
By Mark F. Meier, \\ (U.S. Geological Survey, Tacoma, Washington 98408, U.S.A.) \\ W. Barglay Kamb, Clarence R. Allen and Robert P. Sharp \\ (California Institute of Technology, Pasadena, California 9i Iog, U.S.A.)
}

\begin{abstract}
Velocity and strain-rate patterns in a small temperate valley glacier display flow effects of channel geometry, ice thickness, surface slope, and ablation. Surface velocities of $20-55 \mathrm{~m} /$ year show yearto-year fluctuations of $1.5-3 \mathrm{~m} /$ year. Transverse profiles of velocity have the form of a higher-order parabola modified by the effects of flow around a broad bend in the channel, which makes the velocity profile asymmetric, with maximum velocity displaced toward the outside of the bend. Marginal sliding rates are $5^{-22} \mathrm{~m} /$ year against bedrock and nil against debris. Velocity vectors diverge from the glacier center-line near the terminus, in response to surface ice loss, but converge toward it near the firn line because of channel narrowing. Plunge of the vectors gives an emergence flow component that falls short of balancing ice loss by about I $\mathrm{m} /$ year. Center-line velocities vary systematically with ice thickness and surface slope. In the upper half of the reach studied, effects of changing thickness and slope tend to compensate, and velocities are nearly constant; in the lower half, the effects are cumulative and velocities decrease progressively down-stream. Where the slope increases down-stream from $7^{\circ}$ to $9^{\circ}$, reflecting a bedrock step, there is localized longitudinal extension of 0.03 year $^{-1}$ followed by compression of 0.08 year $^{-1}$ where the slope decreases. Marginal shear (up to 0.5 year $^{-1}$ ) is strongly asymmetric due to flow around the bend: the stress center-line, where one of the principal axes becomes longitudinal, is displaced $150 \mathrm{~m}$ toward the inside of the bend. This effect is prominently visible in the crevasse pattern. Ice fluxes calculated independently by "laminar" flow theory and by continuity disagree in a way which shows that internal deformation of the ice is controlled not by local surface slope but by an effective slope that is nearly constant over the reach studied.
\end{abstract}

RÉsumé. Écoulement du Blue Glacier, Olympic Mountains, Washington, U.S.A. Les comportements de la vitesse et de la vitesse de déformation dans un petit glacier tempéré de vallée reflètent les effets sur l'écoulement de la géométrie du chenal, de l'épaisseur de la neige, de la pente de la surface et de l'ablation. Les vitesses en surface de 20 à $55 \mathrm{~m}$ par an montrent des fluctuations d'une année sur l'autre de 1,5 à $3 \mathrm{~m}$ par an. Les profils transversaux de vitesse ont la forme d'une parabole d'ordre élevé modifiée par les effets de l'écoulement autour d'une large courbure dans le chenal qui rend le profil des vitesses asymétrique avec le maximum de vitesse déplacée vers l'extérieur. Le glissement latéral atteint 5 à $22 \mathrm{~m}$ par an contre la roche en place et s'annule contre les débris. Les vecteurs vitesse divergent depuis la ligne centrale des glaciers près de la langue en réponse à la perte de glace à la surface, mais convergent vers cette ligne centrale près de la ligne des névés à cause du rétrécissement du chenal. Le pendage de ces vecteurs montre une composante remontante de l'écoulement qui ne compense pas, à $1 \mathrm{~m}$ par an près, la perte de glace. Les vitesses à la ligne centrale varient systématiquement avec l'épaisseur de la neige et la pente de la surface. Dans la moitié supérieure du secteur étudié, les effets des changements en épaisseur et en pente tendent à se compenser et les vitesses sont à peu près constantes: dans la moitié inférieure, les effets sont cumulatifs et les vitesses décroissent progressivement de l'amont à l'aval. Lorsque la pente augmente de $7^{\circ}$ à $9^{\circ}$, ce qui reflète une marche dans le lit, il y a une extension longitudinale localisée de $0,03 \mathrm{an}^{-1}$ suivie d'une compression de $0,08 \mathrm{an}^{-1}$ lorsque la pente décroît. Le cisaillement latéral (plus de $0,5 \mathrm{an}^{-1}$ ) est fortement asymétrique en raison de l'écoulement autour de la courbure: la ligne centrale des tensions où l'un des axes principaux devient longitudinal est déplacée de $150 \mathrm{~m}$ vers l'intérieur de la courbe. Cet effet est spécialement visible dans le système de crevasses. Les écoulements de glace calculés indépendamment par la théorie de l'écoulement "laminaire" et par continuité sont en désaccord, ce qui montre que la déformation interne de la glace est réglée non par la pente surperficielle locale mais par une pente moyenne effective qui est à peu près constante sur tout le tronçon étudié.

Zusammenfassung. Das Fliessverhalten des Blue Glacier, Olympic Mountains, Washington, U.S.A. Die Geschwindigkeit und die Spannungsverteilung in einem kleinen, temperierten Talgletscher spiegeln die Fliesseffekte der Gletscherbettgeometrie, der Eisdicke, der Oberflächenneigung und der Ablation wider. Die Oberflächengeschwindigkeiten von $20-55 \mathrm{~m} / \mathrm{a}$ zeigen jährliche Änderungen von $1,5-3 \mathrm{~m} / \mathrm{a}$. Geschwindigkeitsprofile haben die Form einer Parabel höherer Ordnung, modifiziert durch die Auswirkungen einer weiten Kurve des Gletscherbettes, was ein asymmetrisches Geschwindigkeitsprofil zur Folge hat, wobei das Geschwindigkeitsmaximum gegen die Aussenseite der Kurve verschoben ist. Die Gleitgeschwindigkeiten am Rande betragen $5^{-22} \mathrm{~m} / \mathrm{a}$ bei anstehendem Gestein und Null bei Schutt. Die Geschwindigkeitsvektoren divergieren am Gletscherende von der Mittellinie infolge des Eisverlustes an der Oberfläche, konvergieren aber in der Nähe der Firnlinie wegen der Verengung des Gletscherbettes. Das Eintauchen der Vektoren verursacht eine aufsteigende Fliesskomponente, die jedoch den Eisverlust um etwa $\mathrm{i} \mathrm{m} / \mathrm{a}$ nicht ausgleichen kann. Die Geschwindigkeiten längs der Mittellinie schwanken in Abhängigkeit von Eisdicke und Oberflächenneigung

* Contribution No. 2105, Division of Geological and Planetary Sciences, California Institute of Technology. 
systematisch. In der oberen Hälfte des untersuchten Bereiches tendieren die Einflüsse wechselnder Mächtigkeit und Neigung zum Ausgleich, die Geschwindigkeiten sind nahezu konstant; in der unteren Hälfte wirken die Einflüsse kumulativ, die Geschwindigkeiten nehmen gletscherabwärts ständig ab. Wo das Gefälle gletscherabwärts infolge einer Felsschwelle von $7^{\circ}$ auf $9^{\circ}$ zunimmt, tritt lokal eine longitudinale Dehnung von $0,03 \mathrm{a}^{-1}$ auf; es folgt bei wieder geringerem Gefälle Kompression von o,o8 $\mathrm{a}^{-1}$. Wegen des Durchflusses durch die Kurve ist die Randscherung (bis zu o,5 $\mathrm{a}^{-1}$ ) stark asymmetrisch. Die Spannungmittellinie, wo eine der Hauptachsen longitudinal verläuft, ist gegen die Kurveninnenseite um $150 \mathrm{~m}$ verschoben. Das zeigt sich deutlich in der Spaltenanordnung. Die Abweichungen der Eisbewegungen, die unabhängig mit Hilfe der "Laminar"-Fliess-Theorie und der Kontinuitätsbedingung berechnet wurden, zeigen, dass die innere Deformation des Eises nicht von der lokalen Oberflächenneigung, sondern von einer effektiven Neigung abhängt, die im Untersuchungsgebiet beinahe konstant ist.

\section{INTRODUCtion}

Many local measurements of flow velocity have been made on valley glaciers, but only a few studies have provided detailed areal coverage of the velocity field and strain distribution. Movement and strain data are needed for testing theories of glacier flow and response to climatic change. They also aid in the interpretation of glacier structures such as crevasses, foliation, and ogives. In this study of a small valley glacier between firn line and terminus, primary emphasis is on relationships between the velocity field and its controlling factors such as channel geometry, ablation, surface configuration, and velocity boundary conditions (basal and marginal sliding).

Field work for this study began in 1957 and $195^{8}$ as an International Geophysical Year activity, supported by the U.S. National Committee, and continued in 1959 and r 960 with National Science Foundation support. Meier's participation was made possible by the U.S. Geological Survey. Equipment was loaned by the Office of Naval Research (contract N-1896-oo). The National Park Service granted permission for the study, and personnel of Olympic National Park greatly aided the work. Initial supplies and equipment were flown to the glacier by the U.S. Coast Guard and the U.S. Air Force. We are indebted to the University of Washington group operating on the snow dome for much aid. James N. Johnson of the U.S. Geological Survey assisted in the analyses of the strain and velocity data. Many field colleagues, especially C. R. Benson, J. C. Savage, R. L. Shreve, Loren Mosher, and Noel Hinners of the California Institute of Technology, and Dean Johnson of the U.S. Geological Survey gave assistance. The late William R. Fairchild provided superb logistical air support and invaluable help in many ways.

\section{Physical setting}

Blue Glacier is a small valley glacier draining the northeastern slope of Mount Olympus $(2424 \mathrm{~m}$ ) in north-western Washington, U.S.A. It is $4.3 \mathrm{~km}$ long, I $\mathrm{km}$ wide at the firn edge, covers $4.3 \mathrm{~km}^{2}$, and descends from an elevation of $2375 \mathrm{~m}$ to a terminus at $\mathrm{I} 265 \mathrm{~m}$. The firn edge has a mean elevation of about i $600 \mathrm{~m}$, and the bare ice tongue extends roughly $2 \mathrm{~km}$ to a terminus that bifurcates over a longitudinal bedrock ridge. Up-glacier $0.8-0.9 \mathrm{~km}$ from the firn edge is a $300 \mathrm{~m}$ ice fall separating lower Blue Glacier from its principal accumulation basins. Index maps of the glacier, showing the above features, are given by Allen and others (1960, fig. I) and Shreve and Sharp (1970, fig. I). The topography of the part of the glacier studied here is shown in Figure $\mathrm{I}$.

The ice flows in a general northerly direction following a gently curving course, convex to the east over an arc of roughly $60^{\circ}$ with a radius of approximately $\mathrm{I} \mathrm{km} \mathrm{(Fig.} \mathrm{I).} \mathrm{The}$

Fig. I. Surface and bedrock topography of the lower part of Blue Glacier. Ice margin shown stippled. Numbered points are locations of seismic control for bedrock topography. Bore holes are shown with open circles. Bedrock topography is smoothed. Longitudinal and transverse cross-sections (Fig. 2) are located with solid lines. Numbered triangles are surveying instrument stations. 


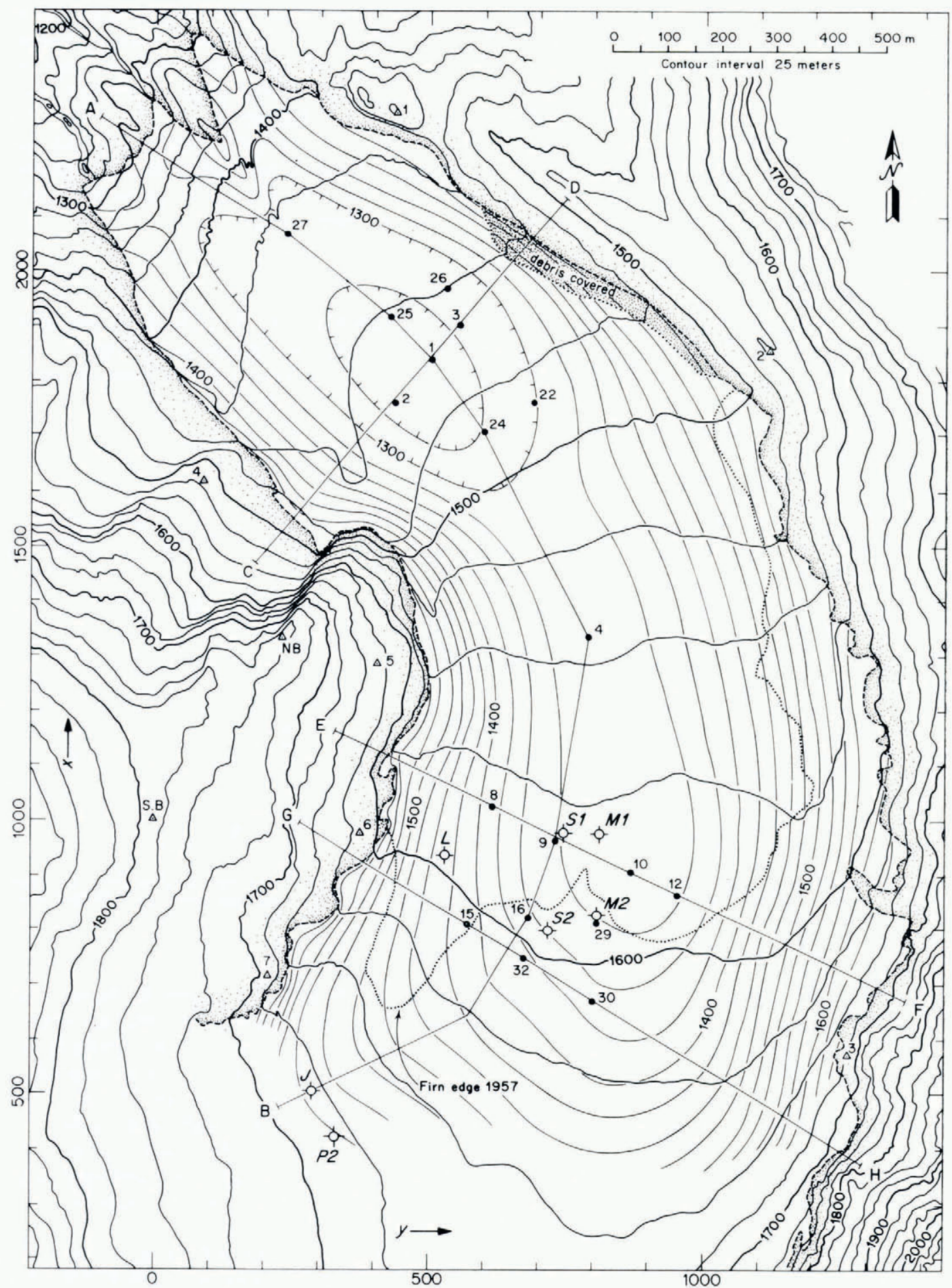

Fig. I. 
surface slope below the firn edge is mostly near $6^{\circ}$. It steepens at the apex of the bend (about $9^{\circ}$ in the middle of the glacier) and near the snout (about $30^{\circ}$ ). Because of marginal rock falls and snow banks, the apparent glacier margin shown on maps does not everywhere correspond precisely with the edge of the flowing ice mass. The flow margin, as indicated by marginal crevasse patterns, is shown as a heavy dashed line in Figure 3 .

The Blue Glacier is a temperate glacier (Harrison, 1972). It has a high degree of activity, judging from its relatively small size and high rates of accumulation and ablation (LaChapelle, I 959, p. 445). The general flow pattern of the glacier, and the relations between flow and internal structure of the ice, are described by Allen and others ( 1960 ).

\section{Bedrock channel configuration}

Seismic reflection shots were made at 33 stations on Blue Glacier. At 18 of these, readable reflections were obtained, and provide the basis for the bedrock contour map (Fig. I) and cross sections (Fig. 2). Although the glacier floor is shown as smooth in the cross-sections,

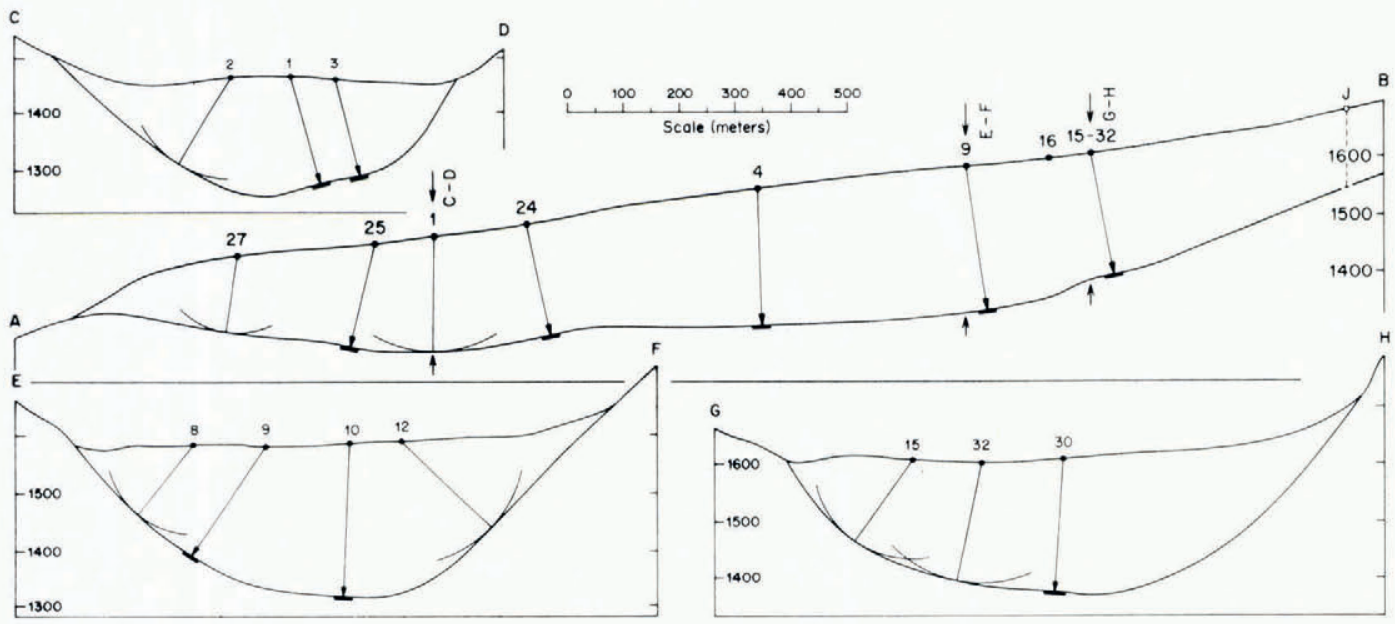

Fig. 2. Longitudinal and transverse cross-sections of Blue Glacier. Locations of sections and numbered control points are given in Figure $I$. Where dip of the bedrock surface could not be determined from seismic reflections, control on the bedrock topography is based on a radius drawn about the shot point as shown.

the subglacial topography is probably of considerable relief, like that presently exposed on the valley walls and below the terminus. On many of the seismic records it is seen that reflections arrived from several directions. Even for very clear reflections, it is often difficult to follow a single reflected phase completely across the geophone spread, or to pick its beginning accurately. Thus we estimate that most of our depth determinations near the center of the glacier are accurate to no more than $10 \mathrm{~m}$, and many near the margins are less accurate.

To determine the seismic velocities, refraction profiles of lengths $68 \mathrm{om}$ and $55^{8} \mathrm{~m}$ were shot across and along the length of the glacier. These profiles were respectively perpendicular and parallel to the predominant foliation in the ice, but both gave velocities close to 3660 $\mathrm{m} / \mathrm{sec}$, with no indication of increase with depth.

The seismic survey confirms the step-like nature of the glacier floor, which can be inferred independently from the surface profile, from the crevasse pattern, and from the results of a gravity study (Corbató, I965). 


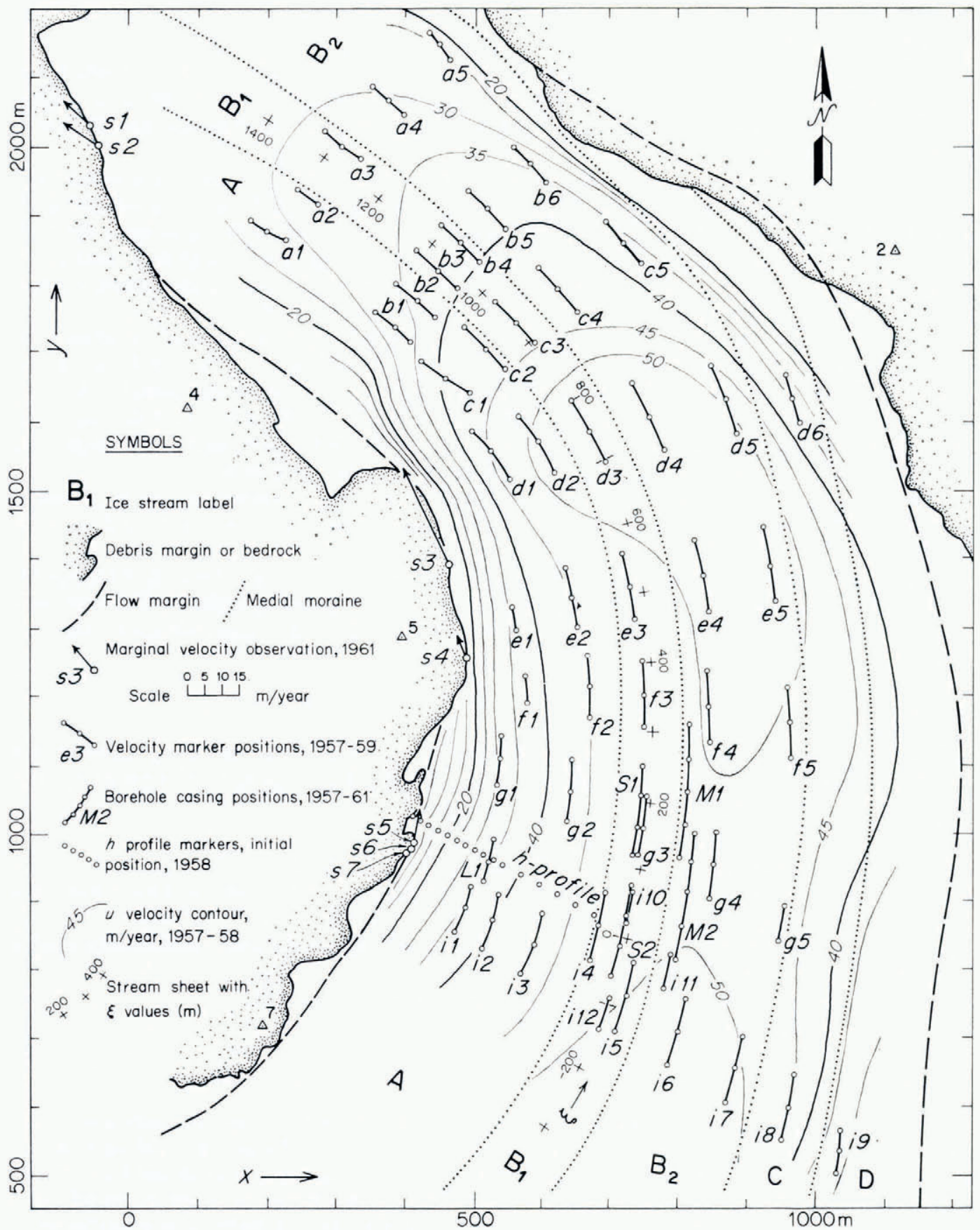

Fig. 3. Location and numbering of velocity markers, showing positions in August 1957, 1958, and 1959. Bore-hole positions in 1960 and 1961 are also shown, Horizontal surface velocity $u$ for $1957-58$ is shown by contours with contour interval $5 \mathrm{~m} /$ year. Marginal sliding velocities in $196 I$ (stations $5 I-57$ ) are plotted at a scale given in the legend. Location of stream sheet followed in flux calculations is shown by train of crosses, with $\xi$ values indicated. 


\section{Surface velocity}

\section{Method of measurement}

Flow velocities were measured by means of marker stakes emplaced in the surface ice along nine transverse profiles across the glacier, as shown in Figure 3. Each transverse profile is designated by a letter (from $a$ to $i$ ) and the individual markers in each profile are identified by a following number (Fig. 3).

The markers were surveyed by triangulation from instrument stations located alongside the glacier (seven numbered triangles in Fig. I). A base line $403.02 \mathrm{~m}$ long was established on bedrock and its length determined by subtense bar. The instrument stations consist of aluminum tubes $0.9 \mathrm{~m}$ long cemented to bedrock (in one instance, stabilized moraine); their coordinates were determined from the base line by a third-order triangulation survey. The surveys utilized Kern DKM 2 or Wild T-2 optical theodolites. *

Three types of markers were used on the ice: 49 consisted of $3.6 \mathrm{~m}$ non-floating aluminum tubes, $3.8 \mathrm{~cm}$ in diameter, closed with a cork or wood dowel at the lower end to prevent melting into the ice. Holes in the ice were drilled $3.1 \mathrm{~m}$ deep using an auger powered by a $2 \frac{1}{2}$ H.P., 2 cycle gasoline engine. 27 other markers consisted of smaller diameter tubes or wood sticks $2 \mathrm{~cm}$ square, emplaced with the aid of the one-inch SIPRE hand auger. The projecting casings of five deep bore holes were also used for velocity measurements.

Marker stakes were reset as ablation required, and were surveyed one or more times during August 1957, 1958, and 1959. The bore-hole casings were also surveyed in i96o and $196 r$.

The measured marker displacements are plotted in Figure 3, and velocity components derived from the displacements for $1957^{-58}$ and $195^{8-59}$ are listed in Table I. The velocities represent averages over the periods of measurement, which are approximately but not exactly one year.

\section{Coordinates}

An $x$-, $y$-, $z$-coordinate system is defined with the $x$-axis directed east, the $y$-axis directed north, and the $z$-axis directed vertically up (Fig. 3). The flow velocity is described in terms of the following components:

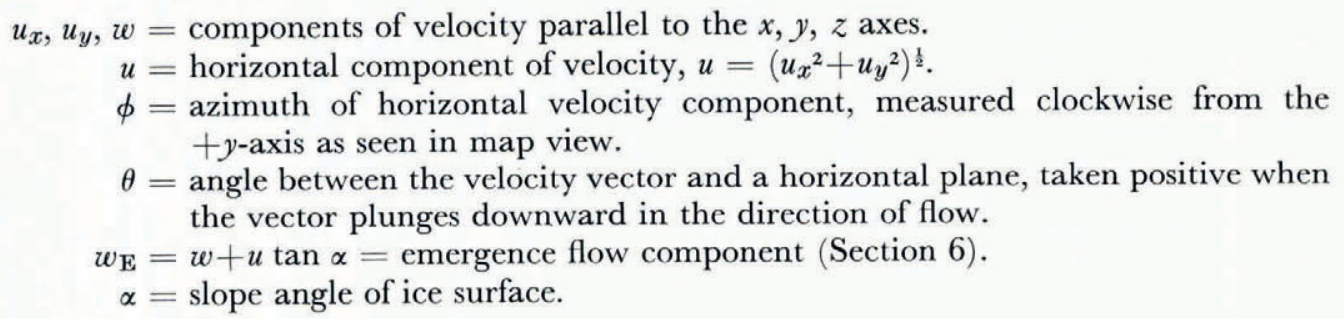

\section{Accuracy}

Errors of closure indicate that the locations of the instrument points are correct to within $3 \mathrm{~cm}$ in a horizontal direction and $7 \mathrm{~cm}$ in a vertical direction. The precision of sighting and reading a surveying instrument and errors caused by varying refraction of the line of eight can be evaluated to some extent by the consistency of locations of markers which were surveyed from more than two instrument points. Differences in horizontal coordinates of stakes revealed by these data averaged $14 \mathrm{~cm}$. The effect of steady refraction is ignored,

* Brand names are given for information only and do not imply endorsement by the U.S. Geological Survey or California Institute of Technology, 
Table I. Velocity components

Upper figures for $1957-58$, lower for ${ }^{1958-59}$

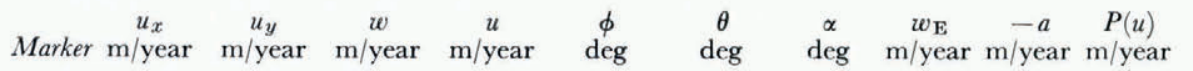

\begin{tabular}{|c|c|c|c|c|c|c|c|c|c|c|c|}
\hline$a I$ & $\begin{array}{l}-23 \cdot 7 \\
-21 \cdot 5\end{array}$ & $\begin{array}{r}+15.3 \\
+16.4\end{array}$ & $\begin{array}{l}-1.5 \\
-0.7\end{array}$ & $\begin{array}{l}+28.1 \\
+27.0\end{array}$ & $\begin{array}{l}-57.1 \\
-52.6\end{array}$ & $\begin{array}{l}3.0 \\
1.4\end{array}$ & $\begin{array}{l}6.3 \\
6.8\end{array}$ & $\begin{array}{r}+1.8 \\
+2.6\end{array}$ & $\begin{array}{l}4 \cdot 1 \\
4 \cdot 3\end{array}$ & $\begin{array}{l}0.4 \\
1.0\end{array}$ & $\begin{array}{l}1.2 \\
1.4\end{array}$ \\
\hline$a 2$ & -25.2 & +20.4 & +1.5 & $+\underline{3}^{2.5}$ & -50.9 & $\begin{array}{l}-2.6 \\
-\end{array}$ & $5 \cdot 7$ & +4.8 & 6. I & 0.4 & 0.7 \\
\hline$a_{3}$ & $\begin{array}{l}-24.8 \\
-25.0\end{array}$ & $\begin{array}{r}+21.5 \\
+20.7\end{array}$ & $\begin{array}{l}+2.5 \\
+0.4\end{array}$ & $\begin{array}{r}+32.8 \\
+32.4\end{array}$ & $\begin{array}{l}-49.0 \\
-50.4\end{array}$ & $\begin{array}{l}-4.4 \\
-0.7\end{array}$ & $\begin{array}{l}6.0 \\
5 \cdot 7\end{array}$ & $\begin{array}{l}+6.2 \\
+3.6\end{array}$ & $\begin{array}{l}3.8 \\
3.2\end{array}$ & $\begin{array}{l}0.4 \\
1.2\end{array}$ & $\begin{array}{l}0.7 \\
0.7\end{array}$ \\
\hline$a_{4}$ & $\begin{array}{l}-23 \cdot 4 \\
-22.4\end{array}$ & $\begin{array}{l}+22.6 \\
+20.2\end{array}$ & $\begin{array}{l}-0.4 \\
+0.7\end{array}$ & $\begin{array}{r}+32.5 \\
+30.2\end{array}$ & $\begin{array}{r}-45.9 \\
-47.9\end{array}$ & $\begin{array}{r}0.6 \\
-1.4\end{array}$ & $\begin{array}{l}4.8 \\
5 \cdot 4\end{array}$ & $\begin{array}{l}+2.6 \\
+3.6\end{array}$ & $\begin{array}{l}2.2 \\
3.4\end{array}$ & $\begin{array}{l}0.2 \\
0.7\end{array}$ & $\begin{array}{l}0.7 \\
1.2\end{array}$ \\
\hline$a_{5}$ & $\begin{array}{l}-13.1 \\
-14.9\end{array}$ & $\begin{array}{l}+22.3 \\
+15.5\end{array}$ & $\begin{array}{l}+0.7 \\
+2.0\end{array}$ & $\begin{array}{r}+25.9 \\
+21.6\end{array}$ & $\begin{array}{l}-30.5 \\
-44.0\end{array}$ & $\begin{array}{l}-1.6 \\
-5.3\end{array}$ & $\begin{array}{l}5 \cdot 5 \\
4 \cdot 9\end{array}$ & $\begin{array}{l}+3.3 \\
+2.0\end{array}$ & $\begin{array}{l}4 \cdot 4 \\
3 \cdot 8\end{array}$ & $\begin{array}{l}0.4 \\
1.9\end{array}$ & $\stackrel{\mathrm{I.2}}{-}$ \\
\hline$b_{I}$ & $\begin{array}{l}-25.2 \\
-26.4\end{array}$ & $\begin{array}{r}+18.3 \\
+20.1\end{array}$ & $\begin{array}{l}+2.2 \\
-0.7\end{array}$ & $\begin{array}{r}+31.0 \\
+33.2\end{array}$ & $\begin{array}{r}-54.1 \\
-52.7\end{array}$ & $\begin{array}{r}-4.0 \\
1.1\end{array}$ & $\begin{array}{l}8.2 \\
6.8\end{array}$ & $\begin{array}{l}+6.6 \\
+3.3\end{array}$ & $\begin{array}{l}5.9 \\
4.0\end{array}$ & $\begin{array}{l}0.2 \\
0.5\end{array}$ & $\begin{array}{l}0.7 \\
0.2\end{array}$ \\
\hline$b_{2}$ & $\begin{array}{l}-27.8 \\
-28.4\end{array}$ & $\begin{array}{r}+23.8 \\
+21.5\end{array}$ & $\begin{array}{l}-1.5 \\
-0.4\end{array}$ & $\begin{array}{r}+36.5 \\
+35.7\end{array}$ & $\begin{array}{r}-49.5 \\
-52.9\end{array}$ & $\begin{array}{l}2.3 \\
0.6\end{array}$ & $\begin{array}{l}6.8 \\
6.8\end{array}$ & $\begin{array}{r}+2.9 \\
+3.9\end{array}$ & $\begin{array}{l}3.1 \\
3.9\end{array}$ & $\begin{array}{l}0.4 \\
0.5\end{array}$ & $\begin{array}{l}1.2 \\
1.2\end{array}$ \\
\hline$b_{3}$ & $\begin{array}{l}-28.1 \\
-29.8\end{array}$ & $\begin{array}{r}+23.8 \\
+27.8\end{array}$ & $\begin{array}{l}-0.7 \\
-1.2\end{array}$ & $\begin{array}{r}+37.6 \\
+40.8\end{array}$ & $\begin{array}{l}-41.6 \\
-46.9\end{array}$ & $\begin{array}{l}\text { I. I } \\
\text { I. } 6\end{array}$ & $\begin{array}{l}6.4 \\
6.3\end{array}$ & $\begin{array}{r}+3.7 \\
+4.4\end{array}$ & $\begin{array}{l}3.8 \\
3.5\end{array}$ & $\begin{array}{l}0.2 \\
0.7\end{array}$ & $\begin{array}{l}0.7 \\
1.2\end{array}$ \\
\hline$b_{4}$ & $\begin{array}{l}-27.8 \\
-26.4\end{array}$ & $\begin{array}{l}+27.8 \\
+24.0\end{array}$ & $\begin{array}{l}-0.2 \\
+0.0+\end{array}$ & $\begin{array}{r}+39.4 \\
+35.6\end{array}$ & $\begin{array}{l}-45.0 \\
-47.7\end{array}$ & $\begin{array}{r}0.3 \\
-0.1\end{array}$ & $\begin{array}{l}6.3 \\
6.0\end{array}$ & $\begin{array}{r}+4.2 \\
+3.7\end{array}$ & $\begin{array}{l}4 \cdot 7 \\
4 \cdot 1\end{array}$ & $\begin{array}{l}0.3 \\
0.3\end{array}$ & $\begin{array}{l}0.7 \\
0.4\end{array}$ \\
\hline$b_{5}$ & $\begin{array}{l}-27.4 \\
-24.9\end{array}$ & $\begin{array}{l}+28.1 \\
+27.2\end{array}$ & $\begin{array}{l}-1.5 \\
-3.5\end{array}$ & $\begin{array}{l}+39.4 \\
+37.2\end{array}$ & $\begin{array}{r}-44.2 \\
-42.4\end{array}$ & $\begin{array}{l}2.1 \\
5 \cdot 4\end{array}$ & $\begin{array}{l}6.2 \\
6.3\end{array}$ & $\begin{array}{r}+2.9 \\
+0.6\end{array}$ & $\begin{array}{l}3 \cdot 4 \\
3 \cdot 9\end{array}$ & $\begin{array}{l}0.2 \\
1.5\end{array}$ & $\begin{array}{l}0.8 \\
1.7\end{array}$ \\
\hline$b 6$ & $\begin{array}{l}-22.6 \\
-23.2\end{array}$ & $\begin{array}{r}+26.6 \\
+ \text { 1 } .6\end{array}$ & $\begin{array}{l}-0.7 \\
-7.1\end{array}$ & $\begin{array}{l}+35.0 \\
+33.0\end{array}$ & $\begin{array}{l}-40.3 \\
-44.6\end{array}$ & $\begin{array}{r}1.2 \\
12.1\end{array}$ & $\begin{array}{l}5 \cdot 7 \\
6.8\end{array}$ & $\begin{array}{l}+2.9 \\
-3.1\end{array}$ & $\underline{4 \cdot 5}$ & $\begin{array}{l}0.4 \\
1.1\end{array}$ & $\begin{array}{l}\text { I. } 2 \\
1.7\end{array}$ \\
\hline$C I$ & $\begin{array}{l}-38.0 \\
-31.6\end{array}$ & $\begin{array}{r}+21.2 \\
+22.9\end{array}$ & $\begin{array}{l}-6.9 \\
-2.5\end{array}$ & $\begin{array}{l}+43.5 \\
+38.8\end{array}$ & $\begin{array}{l}-61.0 \\
-53.9\end{array}$ & $\begin{array}{l}9 \cdot \mathrm{I} \\
3.6\end{array}$ & $\begin{array}{r}11.9 \\
9.9\end{array}$ & $\begin{array}{l}+2.2 \\
+4.3\end{array}$ & $\begin{array}{l}3 \cdot 2 \\
4 \cdot 1\end{array}$ & $\begin{array}{l}0.4 \\
0.3\end{array}$ & $\begin{array}{l}0.7 \\
1.2\end{array}$ \\
\hline$c 2$ & $\begin{array}{l}-28.4 \\
-31.4\end{array}$ & $\begin{array}{r}+29.2 \\
+32.5\end{array}$ & $\begin{array}{l}-6.2 \\
-2.7\end{array}$ & $\begin{array}{r}+40.8 \\
+45.2\end{array}$ & $\begin{array}{r}-44.3 \\
-44.1\end{array}$ & $\begin{array}{l}8.7 \\
2.1\end{array}$ & $\begin{array}{l}9.9 \\
8.0\end{array}$ & $\begin{array}{l}+1.1 \\
+3.6\end{array}$ & $\begin{array}{l}4 \cdot 1 \\
4 \cdot 3\end{array}$ & $\begin{array}{l}\text { I. I } \\
\text { I.I }\end{array}$ & $\begin{array}{r}0.2 \\
1.2\end{array}$ \\
\hline$c_{3}$ & $\begin{array}{l}-28.1 \\
-28.7\end{array}$ & $\begin{array}{r}+32.8 \\
+30.1\end{array}$ & $\begin{array}{l}-5.8 \\
-1.8\end{array}$ & $\begin{array}{r}+43.5 \\
+41.6\end{array}$ & $\begin{array}{l}-40.5 \\
-43.6\end{array}$ & $\begin{array}{l}7 \cdot 7 \\
2.5\end{array}$ & $\begin{array}{l}8.2 \\
7.4\end{array}$ & $\begin{array}{l}+0.4 \\
+3.6\end{array}$ & $\begin{array}{l}4.9 \\
3.8\end{array}$ & $\begin{array}{l}1.0 \\
0.4\end{array}$ & $\begin{array}{l}0.7 \\
1.5\end{array}$ \\
\hline$c_{4}$ & $\begin{array}{l}-25.6 \\
-28.2\end{array}$ & $\begin{array}{r}+34.7 \\
+31.5\end{array}$ & $\begin{array}{l}-6.2 \\
-1.7\end{array}$ & $\begin{array}{l}+43.0 \\
+42.2\end{array}$ & $\begin{array}{l}-36.4 \\
-41.8\end{array}$ & $\begin{array}{l}8.2 \\
2.3\end{array}$ & $\begin{array}{l}7 \cdot 7 \\
7.1\end{array}$ & $\begin{array}{l}-0.4 \\
+3.5\end{array}$ & $\begin{array}{l}4.8 \\
3.8\end{array}$ & $\begin{array}{l}0.2 \\
0.5\end{array}$ & $\begin{array}{l}0.2 \\
1.0\end{array}$ \\
\hline$c_{5}$ & $\begin{array}{l}-24.8 \\
-24.3\end{array}$ & $\begin{array}{l}+28.4 \\
+26.8\end{array}$ & $\begin{array}{l}+0.7 \\
+2.9\end{array}$ & $\begin{array}{r}+38.0 \\
+3^{6.2}\end{array}$ & $\begin{array}{r}-49.1 \\
-42.3\end{array}$ & $\begin{array}{l}1.1 \\
5.1\end{array}$ & $\begin{array}{l}7 \cdot 3 \\
7 \cdot 4\end{array}$ & $\begin{array}{l}+5.5 \\
+1.8\end{array}$ & $\begin{array}{l}4.1 \\
4.0\end{array}$ & $\begin{array}{l}0.4 \\
1.0\end{array}$ & $\begin{array}{l}1.2 \\
1.7\end{array}$ \\
\hline$d I$ & $\begin{array}{l}-27.6 \\
-26.3\end{array}$ & $\begin{array}{r}+39.8 \\
+27.6\end{array}$ & $\begin{array}{r}-17.2 \\
-10.5\end{array}$ & $\begin{array}{r}+47.8 \\
+38.2\end{array}$ & $\begin{array}{l}-33.8 \\
-43.6\end{array}$ & $\begin{array}{l}19.7 \\
15.5\end{array}$ & $\begin{array}{l}\text { I } 6.6 \\
\text { I } 5.1\end{array}$ & $\begin{array}{l}-2.9 \\
-0.3\end{array}$ & $\begin{array}{l}2.6 \\
3 \cdot 4\end{array}$ & $\begin{array}{l}0.2 \\
0.3\end{array}$ & $\begin{array}{l}0.2 \\
1.1\end{array}$ \\
\hline$d 2$ & $\begin{array}{l}-24.8 \\
-27.2\end{array}$ & $\begin{array}{l}+44.5 \\
+39.6\end{array}$ & $\begin{array}{l}-8.8 \\
-7.6\end{array}$ & $\begin{array}{r}+51.0 \\
+44.1\end{array}$ & $\begin{array}{l}-29.1 \\
-38.2\end{array}$ & $\begin{array}{l}9.8 \\
9.8\end{array}$ & $\begin{array}{l}11.6 \\
11.6\end{array}$ & $\begin{array}{r}+1.8 \\
+1.4\end{array}$ & $\begin{array}{l}2.6 \\
3 \cdot 1\end{array}$ & $\begin{array}{l}0.4 \\
0.3\end{array}$ & $\begin{array}{l}0.8 \\
0.9\end{array}$ \\
\hline$d_{3}$ & $\begin{array}{l}-23.4 \\
-25.8\end{array}$ & $\begin{array}{r}+45.6 \\
+41.2\end{array}$ & $\begin{array}{l}-5.5 \\
-6.1\end{array}$ & $\begin{array}{r}+5^{1} .5 \\
+4^{8.4}\end{array}$ & $\begin{array}{l}-27.1 \\
-28.8\end{array}$ & $\begin{array}{l}6.1 \\
7.2\end{array}$ & $\begin{array}{l}8.9 \\
9 \cdot 9\end{array}$ & $\begin{array}{l}+2.6 \\
+2.4\end{array}$ & $\begin{array}{l}3 \cdot 0 \\
3 \cdot 3\end{array}$ & $\begin{array}{l}0.6 \\
0.2\end{array}$ & $\begin{array}{l}0.9 \\
0.7\end{array}$ \\
\hline$d_{4}$ & $\begin{array}{l}-22.6 \\
-24.6\end{array}$ & $\begin{array}{r}+48.9 \\
+44.6\end{array}$ & $\begin{array}{l}-5 \cdot 1 \\
-5 \cdot 4\end{array}$ & $\begin{array}{r}+54.0 \\
+51.0\end{array}$ & $\begin{array}{l}-24.8 \\
-28.8\end{array}$ & $\begin{array}{l}5 \cdot 4 \\
6.0\end{array}$ & $\begin{array}{l}7.4 \\
8.8\end{array}$ & $\begin{array}{l}+1.8 \\
+2.5\end{array}$ & $\begin{array}{l}2.7 \\
3.6\end{array}$ & $\begin{array}{l}0.4 \\
0.2\end{array}$ & $\begin{array}{l}0.7 \\
0.7\end{array}$ \\
\hline$d_{5}$ & $\begin{array}{l}-19.0 \\
-22.1\end{array}$ & $\begin{array}{r}+46.4 \\
+44.9\end{array}$ & $\begin{array}{l}-1.1 \\
-2.9\end{array}$ & $\begin{array}{r}+50.0 \\
+50.0\end{array}$ & $\begin{array}{l}-22.3 \\
-26.2\end{array}$ & $\begin{array}{l}\text { I. } 3 \\
3 \cdot 3\end{array}$ & $\begin{array}{l}7 \cdot 5 \\
7 \cdot 1\end{array}$ & $\begin{array}{r}+5.5 \\
+3.4\end{array}$ & $\begin{array}{l}6.1 \\
4.0\end{array}$ & $\begin{array}{l}0.5 \\
1.0\end{array}$ & $\begin{array}{l}1.2 \\
1.7\end{array}$ \\
\hline$d 6$ & $\begin{array}{r}-9.9 \\
- \text { I0.0 }\end{array}$ & $\begin{array}{l}+32.8 \\
+30.3\end{array}$ & $\begin{array}{l}-1.8 \\
+1.0\end{array}$ & $\begin{array}{l}+34.3 \\
+32.0\end{array}$ & $\begin{array}{l}-16.7 \\
-18.3\end{array}$ & $\begin{array}{r}3.0 \\
-1.8\end{array}$ & $\begin{array}{l}6.4 \\
6.3\end{array}$ & $\begin{array}{l}+2.2 \\
+2.5\end{array}$ & $\begin{array}{l}3.0 \\
3.9\end{array}$ & $\begin{array}{l}0.4 \\
1.1\end{array}$ & $\begin{array}{l}1.2 \\
1.8\end{array}$ \\
\hline$e I$ & $-5 \cdot 1$ & $+\underline{35.4}$ & -4.4 & +35.8 & $\begin{array}{l}-8.2 \\
-\end{array}$ & $\underbrace{7.0}$ & $\frac{9.8}{-}$ & +1.8 & $\begin{array}{l}3 \cdot 4 \\
4 \cdot 2\end{array}$ & $\underline{0.4}$ & 0.7 \\
\hline$e 2$ & $\begin{array}{l}-7.3 \\
-9.6\end{array}$ & $\begin{array}{r}+43.8 \\
+39.4\end{array}$ & $\begin{array}{l}-4.7 \\
-5.6\end{array}$ & $\begin{array}{l}+44.5 \\
+39.2\end{array}$ & $\begin{array}{r}-9.5 \\
-\mathbf{r} 3.6\end{array}$ & $\begin{array}{l}6.1 \\
8.2\end{array}$ & $\begin{array}{l}8.3 \\
8.0\end{array}$ & $\begin{array}{l}+1.8 \\
+0.1\end{array}$ & $\begin{array}{l}2.3 \\
2.8\end{array}$ & $\begin{array}{l}0.4 \\
0.2\end{array}$ & $\begin{array}{l}1.2 \\
0.7\end{array}$ \\
\hline e3 & $\begin{array}{r}-8.8 \\
-10.7\end{array}$ & $\begin{array}{r}+48.2 \\
+45.4\end{array}$ & $\begin{array}{l}-3.6 \\
-3.8\end{array}$ & $\begin{array}{r}+48.5 \\
+47.5\end{array}$ & $\begin{array}{l}-10.3 \\
-13.1\end{array}$ & $\begin{array}{l}4 \cdot 3 \\
4.6\end{array}$ & $\begin{array}{l}6.9 \\
7.4\end{array}$ & $\begin{array}{l}+2.2 \\
+2.2\end{array}$ & $\begin{array}{l}2.4 \\
2.7\end{array}$ & $\begin{array}{l}0.3 \\
0.2\end{array}$ & $\begin{array}{l}0.9 \\
0.8\end{array}$ \\
\hline
\end{tabular}


TABle I - continued

\begin{tabular}{|c|c|c|c|c|c|c|c|c|c|c|c|}
\hline $\begin{array}{c}u_{x} \\
\mathrm{~m} / \text { year }\end{array}$ & $\begin{array}{c}u_{y} \\
\mathrm{~m} / \text { year }\end{array}$ & $\begin{array}{c}w \\
\mathrm{~m} / \text { year }\end{array}$ & $\begin{array}{c}u \\
\mathrm{~m} / \text { year }\end{array}$ & $\stackrel{\phi}{\operatorname{deg}}$ & $\stackrel{\theta}{\operatorname{deg}}$ & $\stackrel{\alpha}{\operatorname{deg}}$ & $\begin{array}{c}w_{\mathrm{E}} \\
\mathrm{m} / \text { year }\end{array}$ & $\begin{array}{c}-a \\
\mathrm{~m} / \text { year }\end{array}$ & $\begin{array}{c}P(u) \\
\text { m/year } \\
\pm\end{array}$ & \multicolumn{2}{|c|}{$\begin{array}{c}P(w) \\
\text { m/year } \\
+\quad\end{array}$} \\
\hline $\begin{array}{r}-9.5 \\
- \text { I I.9 }\end{array}$ & $\begin{array}{r}+51.8 \\
+48.7\end{array}$ & $\begin{array}{l}-3.6 \\
-2.3\end{array}$ & $\begin{array}{r}+52.9 \\
+50.2\end{array}$ & $\begin{array}{l}-10.4 \\
-13.6\end{array}$ & $\begin{array}{l}4.0 \\
2.7\end{array}$ & $\begin{array}{l}6.2 \\
6.8\end{array}$ & $\begin{array}{l}+2.2 \\
+3.7\end{array}$ & $\begin{array}{l}2.8 \\
3 \cdot 3\end{array}$ & $\begin{array}{l}0.4 \\
0.2\end{array}$ & $\begin{array}{l}1.3 \\
0.8\end{array}$ & $\begin{array}{l}0.3 \\
0.3\end{array}$ \\
\hline $\begin{array}{r}-10.2 \\
-7.4\end{array}$ & $\begin{array}{r}+50.3 \\
+55.5\end{array}$ & $\begin{array}{l}-2.6 \\
-3.6\end{array}$ & $\begin{array}{r}+51.4 \\
+55.9\end{array}$ & $\begin{array}{r}-11.5 \\
-7.6\end{array}$ & $\begin{array}{l}2.8 \\
3.7\end{array}$ & $\begin{array}{l}6.0 \\
7 \cdot 4\end{array}$ & $\begin{array}{l}+2.9 \\
+3.7\end{array}$ & $\begin{array}{l}3 \cdot 3 \\
4 \cdot 4\end{array}$ & $\begin{array}{l}0.4 \\
2.2\end{array}$ & $\begin{array}{l}1.2 \\
2.5\end{array}$ & $\begin{array}{l}0.2 \\
1.0\end{array}$ \\
\hline $\begin{array}{l}+0.2 \\
-\end{array}$ & $+\underline{3}^{8.7}$ & -2.9 & $+\underline{38.7}$ & +0.3 & $4 \cdot 3$ & 6.I & +1.1 & 3.8 & $\frac{0.4}{-}$ & $\frac{1.2}{-}$ & $\stackrel{0.2}{-}$ \\
\hline $\begin{array}{l}-0.7 \\
-2.3\end{array}$ & $\begin{array}{r}+45.3 \\
+43.7\end{array}$ & $\begin{array}{l}-3 \cdot 3 \\
-3 \cdot 5\end{array}$ & $\begin{array}{r}+45.5 \\
+43.7\end{array}$ & $\begin{array}{l}-0.9 \\
-3.0\end{array}$ & $\begin{array}{l}4 \cdot 1 \\
4 \cdot 5\end{array}$ & $\begin{array}{l}6.4 \\
7 \cdot 7\end{array}$ & $\begin{array}{r}+1.8 \\
+2.4\end{array}$ & $\begin{array}{l}2.1 \\
2.7\end{array}$ & $\begin{array}{l}0.2 \\
0.2\end{array}$ & $\begin{array}{l}0.7 \\
0.7\end{array}$ & $\begin{array}{l}0.2 \\
0.2\end{array}$ \\
\hline $\begin{array}{l}-0.3 \\
-2.9\end{array}$ & $\begin{array}{r}+49.2 \\
+47.9\end{array}$ & $\begin{array}{l}-3.6 \\
-3.2\end{array}$ & $\begin{array}{r}+49.2 \\
+48.0\end{array}$ & $\begin{array}{l}-0.3 \\
-3.5\end{array}$ & $\begin{array}{l}4.2 \\
3.6\end{array}$ & $\begin{array}{l}5 \cdot 7 \\
6.8\end{array}$ & $\begin{array}{l}+1.5 \\
+2.5\end{array}$ & $\begin{array}{l}1.9 \\
2.7\end{array}$ & $\begin{array}{l}0.2 \\
0.2\end{array}$ & $\begin{array}{l}0.7 \\
0.9\end{array}$ & $\begin{array}{l}0.2 \\
0.4\end{array}$ \\
\hline $\begin{array}{l}-0.7 \\
-2.8\end{array}$ & $\begin{array}{r}+51.5 \\
+49.5\end{array}$ & $\begin{array}{l}-3.6 \\
-3.0\end{array}$ & $\begin{array}{r}+51.5 \\
+49.6\end{array}$ & $\begin{array}{l}-0.8 \\
-3 \cdot 3\end{array}$ & $\begin{array}{l}4 \cdot 1 \\
3 \cdot 4\end{array}$ & $\begin{array}{l}5 \cdot 3 \\
6.3\end{array}$ & $\begin{array}{l}+\mathrm{I} . \mathrm{I} \\
+2.5\end{array}$ & $\begin{array}{l}2.3 \\
2.8\end{array}$ & $\begin{array}{l}0.2 \\
0.2\end{array}$ & $\begin{array}{l}0.7 \\
\text { I. }\end{array}$ & $\begin{array}{l}0.2 \\
0.5\end{array}$ \\
\hline $\begin{array}{l}-0.4 \\
-2.9\end{array}$ & $\begin{array}{r}+50.7 \\
+49.0\end{array}$ & $\begin{array}{l}-2.2 \\
-2.2\end{array}$ & $\begin{array}{r}+50.7 \\
+49.2\end{array}$ & $\begin{array}{l}-0.4 \\
-3.4\end{array}$ & $\begin{array}{l}2.5 \\
2.6\end{array}$ & $\begin{array}{l}5 \cdot 1 \\
5 \cdot 7\end{array}$ & $\begin{array}{l}+2.2 \\
+2.7\end{array}$ & $\begin{array}{l}3 \cdot 9 \\
4.0\end{array}$ & $\begin{array}{l}0.4 \\
1.0\end{array}$ & $\begin{array}{l}1.2 \\
1.5\end{array}$ & $\begin{array}{l}0.2 \\
0.4\end{array}$ \\
\hline $\begin{array}{l}+2.9 \\
+2.6\end{array}$ & $\begin{array}{r}+31.4 \\
+29.9\end{array}$ & $\begin{array}{l}-0.4 \\
+0.9\end{array}$ & $\begin{array}{r}+31.4 \\
+30.0\end{array}$ & $\begin{array}{r}+5.3 \\
+4.9\end{array}$ & $\begin{array}{l}0.7 \\
1.7\end{array}$ & $\begin{array}{l}4 \cdot 5 \\
5 \cdot 4\end{array}$ & $\begin{array}{l}+2.2 \\
+3.7\end{array}$ & $\begin{array}{l}2.5 \\
4 \cdot \mathrm{I}\end{array}$ & $\begin{array}{l}0.2 \\
0.3\end{array}$ & $\begin{array}{l}0.7 \\
0.9\end{array}$ & $\begin{array}{l}0.2 \\
0.2\end{array}$ \\
\hline $\begin{array}{l}+4.4 \\
+5.0\end{array}$ & $\begin{array}{r}+43.0 \\
+42.6\end{array}$ & $\begin{array}{l}-2.2 \\
-\mathrm{I} . \mathrm{I}\end{array}$ & $\begin{array}{r}+43.4 \\
+42.8\end{array}$ & $\begin{array}{r}+5.8 \\
+6.7\end{array}$ & $\begin{array}{r}2.9 \\
-\mathrm{I} .5\end{array}$ & $\begin{array}{l}5.0 \\
5.1\end{array}$ & $\begin{array}{l}+1.5 \\
+2.8\end{array}$ & $\begin{array}{l}1.6 \\
2.8\end{array}$ & $\begin{array}{l}0.2 \\
0.2\end{array}$ & $\begin{array}{l}0.7 \\
0.8\end{array}$ & $\begin{array}{l}0.2 \\
0.3\end{array}$ \\
\hline $\begin{array}{l}+6.9 \\
+4.9\end{array}$ & $\begin{array}{r}+48.5 \\
+45.5\end{array}$ & $\begin{array}{l}-0.0- \\
-\mathrm{I} .4\end{array}$ & $\begin{array}{r}+48.9 \\
+45.8\end{array}$ & $\begin{array}{l}+8.1 \\
+6.1\end{array}$ & $\begin{array}{l}0.0 \\
\text { I.7 }\end{array}$ & $\begin{array}{l}4.7 \\
4.6\end{array}$ & $\begin{array}{l}+4.0 \\
+2.3\end{array}$ & $\begin{array}{l}4.2 \\
2.6\end{array}$ & $\begin{array}{l}0.5 \\
0.5\end{array}$ & $\begin{array}{l}0.9 \\
0.9\end{array}$ & $\begin{array}{l}0.6 \\
0.4\end{array}$ \\
\hline $\begin{array}{l}+6.6 \\
+5.4\end{array}$ & $\begin{array}{r}+50.0 \\
+47.4\end{array}$ & $\begin{array}{l}-0.7 \\
-0.9\end{array}$ & $\begin{array}{r}+50.4 \\
+47.6\end{array}$ & $\begin{array}{r}+7.5 \\
+6.5\end{array}$ & $\begin{array}{l}0.8 \\
\text { I. I }\end{array}$ & $\begin{array}{l}4 \cdot 5 \\
4 \cdot 5\end{array}$ & $\begin{array}{r}+3.3 \\
+2.9\end{array}$ & $\begin{array}{l}1.5 \\
2.7\end{array}$ & $\begin{array}{l}0.3 \\
0.2\end{array}$ & $\begin{array}{l}0.8 \\
0.9\end{array}$ & $\begin{array}{l}0.3 \\
0.4\end{array}$ \\
\hline+6.9 & $+\underline{47.8}$ & -0.2 & $+\underline{48.1}$ & +8.3 & 0.3 & $\frac{4 \cdot 3}{-}$ & $+3 \cdot 4$ & $4 \cdot 5$ & 0.5 & 1.3 & $\underline{0.3}$ \\
\hline $\begin{array}{r}+12.0 \\
+10.5\end{array}$ & $\begin{array}{l}+34.6 \\
+30.6\end{array}$ & $\begin{array}{l}-4.7 \\
-6.8\end{array}$ & $\begin{array}{r}+35.8 \\
+32.4\end{array}$ & $\begin{array}{r}+19.7 \\
+19.0\end{array}$ & $\begin{array}{r}7.6 \\
11.9\end{array}$ & $\begin{array}{r}10.7 \\
9.6\end{array}$ & $\begin{array}{l}+2.2 \\
-\mathrm{I} .3\end{array}$ & $\frac{2.6}{-}$ & $\begin{array}{l}0.4 \\
0.2\end{array}$ & $\begin{array}{l}0.7 \\
0.2\end{array}$ & $\begin{array}{l}0.2 \\
0.2\end{array}$ \\
\hline $\begin{array}{r}+14.2 \\
+10.9\end{array}$ & $\begin{array}{r}+38.7 \\
+35.6\end{array}$ & $\begin{array}{l}-6.6 \\
-3.9\end{array}$ & $\begin{array}{r}+41.2 \\
+37.8\end{array}$ & $\begin{array}{r}+20.2 \\
+17.1\end{array}$ & $\begin{array}{l}9 \cdot 1 \\
5 \cdot 9\end{array}$ & $\begin{array}{l}8.9 \\
8.0\end{array}$ & $\begin{array}{l}+0.0 \\
+1.4\end{array}$ & 2.5 & $\begin{array}{l}0.4 \\
0.2\end{array}$ & $\begin{array}{l}1.2 \\
0.7\end{array}$ & $\begin{array}{l}0.2 \\
0.2\end{array}$ \\
\hline $\begin{array}{l}+ \text { r } 6.8 \\
+ \text { r } 2.6\end{array}$ & $\begin{array}{r}+39.8 \\
+41.5\end{array}$ & $\begin{array}{l}-5.1 \\
-4.7\end{array}$ & $\begin{array}{l}+43.0 \\
+43.5\end{array}$ & $\begin{array}{r}+22.9 \\
+16.9\end{array}$ & $\begin{array}{l}6.7 \\
6.2\end{array}$ & $\begin{array}{l}8.2 \\
6.8\end{array}$ & $\begin{array}{l}+1.1 \\
+0.5\end{array}$ & $\frac{2 \cdot 3}{-}$ & $\begin{array}{l}0.4 \\
0.2\end{array}$ & $\begin{array}{l}1.2 \\
0.7\end{array}$ & $\begin{array}{l}0.2 \\
0.2\end{array}$ \\
\hline $\begin{array}{l}+12.0 \\
+12.0\end{array}$ & $\begin{array}{l}+47.8 \\
+46.5\end{array}$ & $\begin{array}{l}-3.6 \\
-4.0\end{array}$ & $\begin{array}{r}+49.2 \\
+48.0\end{array}$ & $\begin{array}{r}+14.1 \\
+14.5\end{array}$ & $\begin{array}{l}4 \cdot 2 \\
4 \cdot 7\end{array}$ & $\begin{array}{l}5 \cdot 9 \\
5 \cdot 1\end{array}$ & $\begin{array}{l}+1.5 \\
+0.4\end{array}$ & $\begin{array}{l}2.8 \\
2.7\end{array}$ & $\begin{array}{l}0.4 \\
0.9\end{array}$ & $\begin{array}{l}0.7 \\
0.5\end{array}$ & $\begin{array}{l}0.2 \\
0.5\end{array}$ \\
\hline $\begin{array}{r}+16.8 \\
+13.6\end{array}$ & $\begin{array}{r}+49.2 \\
+45.6\end{array}$ & $\begin{array}{l}-6.2 \\
-4.1\end{array}$ & $\begin{array}{r}+52.1 \\
+47.6\end{array}$ & $\begin{array}{r}+18.8 \\
+16.7\end{array}$ & $\begin{array}{l}6.8 \\
4.9\end{array}$ & $\begin{array}{l}8.2 \\
6.0\end{array}$ & $\begin{array}{l}+1.5 \\
+1.0\end{array}$ & $\begin{array}{l}2.1 \\
2.5\end{array}$ & $\begin{array}{l}0.4 \\
0.4\end{array}$ & $\begin{array}{l}1.2 \\
0.7\end{array}$ & $\begin{array}{l}0.2 \\
0.2\end{array}$ \\
\hline $\begin{array}{r}+15.0 \\
+12.4\end{array}$ & $\begin{array}{r}+49.6 \\
+46.2\end{array}$ & $\begin{array}{l}-6.6 \\
-4.7\end{array}$ & $\begin{array}{r}+51.8 \\
+47.9\end{array}$ & $\begin{array}{r}+16.8 \\
+15.0\end{array}$ & $\begin{array}{l}7 \cdot 2 \\
5 \cdot 7\end{array}$ & $\begin{array}{l}7.2 \\
6.0\end{array}$ & $\begin{array}{r}0.0 \\
+0.3\end{array}$ & $\begin{array}{l}\text { 1.0 } \\
1.8\end{array}$ & $\begin{array}{l}0.4 \\
0.6\end{array}$ & $\begin{array}{l}0.7 \\
0.5\end{array}$ & $\begin{array}{l}0.2 \\
0.5\end{array}$ \\
\hline $\begin{array}{r}+13.5 \\
+11.2\end{array}$ & $\begin{array}{r}+48.9 \\
+45.4\end{array}$ & $\begin{array}{l}-5.1 \\
-4.1\end{array}$ & $\begin{array}{r}+50.7 \\
+46.7 \\
\end{array}$ & $\begin{array}{l}+15.4 \\
+13.9\end{array}$ & $\begin{array}{l}5.8 \\
5.0\end{array}$ & $\begin{array}{l}5 \cdot 7 \\
5 \cdot 4\end{array}$ & $\begin{array}{r}0.0 \\
+0.3\end{array}$ & $\begin{array}{l}2.3 \\
2.2\end{array}$ & $\begin{array}{l}0.3 \\
0.3\end{array}$ & $\begin{array}{l}0.8 \\
0.8\end{array}$ & $\begin{array}{l}0.3 \\
0.3\end{array}$ \\
\hline $\begin{array}{l}+11.7 \\
+10.2\end{array}$ & $\begin{array}{r}+44.1 \\
+42.0\end{array}$ & $\begin{array}{l}-4.4 \\
-4.2\end{array}$ & $\begin{array}{r}+45.6 \\
+43.2\end{array}$ & $\begin{array}{r}+14.8 \\
+13.7\end{array}$ & $\begin{array}{l}5 \cdot 5 \\
5 \cdot 5\end{array}$ & $\begin{array}{l}5.8 \\
6.3\end{array}$ & $\begin{array}{l}+0.4 \\
+0.6\end{array}$ & $\begin{array}{l}2.4 \\
2.7\end{array}$ & $\begin{array}{l}0.4 \\
0.6\end{array}$ & $\begin{array}{l}1.3 \\
0.8\end{array}$ & $\begin{array}{l}0.3 \\
0.3\end{array}$ \\
\hline $\begin{array}{r}+5.1 \\
+3.0\end{array}$ & $\begin{array}{r}+30.3 \\
+26.9\end{array}$ & $\begin{array}{l}-3.3 \\
-6.9\end{array}$ & $\begin{array}{l}+30.6 \\
+27.0\end{array}$ & $\begin{array}{r}+9.6 \\
+6.3\end{array}$ & $\begin{array}{r}6.1 \\
\text { I } 4.4\end{array}$ & $\begin{array}{l}5.9 \\
6.0\end{array}$ & $\begin{array}{l}-0.4 \\
-4.1\end{array}$ & $-2 \cdot 3$ & $\begin{array}{l}0.5 \\
1.1\end{array}$ & $\begin{array}{l}0.8 \\
2.4\end{array}$ & $\begin{array}{l}0.3 \\
0.4\end{array}$ \\
\hline$+\overline{10.2}$ & $-\overline{45 \cdot 4}$ & -2.3 & $+\overline{46.5}$ & $+\overline{12.7}$ & $\overline{2.8}$ & $\overline{5 \cdot 1}$ & $\overline{+1} \cdot 9$ & - & $\overline{0.2}$ & $\overline{0.2}$ & $\overline{0.2}$ \\
\hline$+\overline{10.7}$ & $+\overline{48.8}$ & $-5 \cdot 3$ & $+\overline{50.0}$ & $+\overline{12.3}$ & $\overline{6 . I}$ & $\overline{4.9}$ & $-I^{-I}$ & $\overline{1.4}$ & $\overline{0.6}$ & $\overline{0.3}$ & $\overline{0.3}$ \\
\hline$+\overline{16.3}$ & $\begin{array}{c}- \\
+45.2\end{array}$ & -5.1 & $+\overline{48.0}$ & $+\overline{\text { I } 9.8}$ & $\overline{6 . \mathrm{I}}$ & $\overline{7.1}$ & 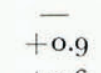 & $\overline{2.2}$ & $\overline{0.3}$ & $\overline{0.6}$ & $\overline{0.6}$ \\
\hline $\begin{array}{r}+5.8 \\
+4.3\end{array}$ & $\begin{array}{r}+50.4 \\
+47.6\end{array}$ & $\begin{array}{l}+1.1 \\
+1.9\end{array}$ & $\begin{array}{r}+50.7 \\
+47.8\end{array}$ & $\begin{array}{l}+6.6 \\
+5.1\end{array}$ & $\begin{array}{l}-1.2 \\
-2.3\end{array}$ & $\begin{array}{l}4.0 \\
4.6\end{array}$ & $\begin{array}{l}+2.6 \\
+5.7\end{array}$ & - & - & - & 二 \\
\hline $\begin{array}{r}+9.8 \\
+8.3\end{array}$ & $\begin{array}{r}+49.2 \\
+46.3\end{array}$ & $\begin{array}{l}-5.5 \\
-0.4\end{array}$ & $\begin{array}{r}+50.4 \\
+46.0\end{array}$ & $\begin{array}{l}+11.3 \\
+10.1\end{array}$ & $\begin{array}{l}6.2 \\
0.5\end{array}$ & $\begin{array}{l}4 \cdot 9 \\
4 \cdot 3\end{array}$ & $\begin{array}{l}-1.1 \\
+3.1\end{array}$ & - & - & - & - \\
\hline$\overline{+6.3}$ & $\overline{+45.4}$ & $\overline{-3.1}$ & $+\overline{45.7}$ & $\overline{+7.9}$ & $\overline{3.9}$ & $\overline{4.6}$ & - & - & - & - & - \\
\hline
\end{tabular}


because only changes in apparent stake location were used to compute velocity components. Errors due to resetting of stakes, and the wobbling, leaning, and settling of stakes were estimated in the field. Estimated probable errors for $u$ and $w$, combined from the various sources mentioned, are reported as $P(u)$ and $P(w)$ for each marker in Table I. The asymmetry in $P(w)$ arises from leaning and settling of stakes; the resulting errors that would algebraically decrease $w$ tend to be larger than those that would increase $w$. Taking into account these asymmetric errors, the permissible range of $w$ is obtained from the measured $w$ values in Table I by adding and subtracting the separate $\pm P(w)$ values as indicated.

\section{Time variations of velocity}

Velocities at most of the markers for $195^{8-59}$ were less by $\mathrm{I}-4 \mathrm{~m}^{-}$year $^{-1}$ than those for I $957-58$. For the $a-c$ profiles the decrease (averaging I. I $\mathrm{m} \mathrm{year}^{-1}$ ) is attributable mainly to motion of the markers through a longitudinally varying velocity field. Above the $d$ profile, where longitudinal gradients are small, the decrease represents a time variation in the velocity field. Time variations over the period $1957-6$ I were shown by the bore-hole casings, which were moving in an area where longitudinal variations in velocity were small and well defined (Fig. 3). Compared with the $1957^{-58}$ velocity field, the $195^{8-} 59$ velocities of these markers were low by about $3 \mathrm{~m}_{\text {year }}^{-\mathrm{I}}$, the $1959-60$ velocities low by about $\mathrm{I} .5 \mathrm{~m}$ year ${ }^{-1}$, and the I960-6r velocities high by about $2 \mathrm{~m}_{\text {year }}{ }^{-1}$.

Three markers showed substantial increases in velocity from $1957-5^{8}$ to $195^{8-59}\left(b_{3}, c 2, e_{5}\right.$, Table I); these were localized at the individual markers and do not conform to the general pattern observed.

Year-to-year velocity fluctuations of the magnitude observed could be caused by changes in ice thickness and surface slope. From glacier flow theory, a decrease in ice thickness of I $\mathrm{m}$ should reduce $u$ by $0.7^{-1} .4 \mathrm{~m}$ year $^{-1}$, and a decrease in surface slope of $0.05^{\circ}$ (which would lower the ice $0.5 \mathrm{~m}$ less at the $e$ profile than at the $i$ profile) should reduce $u$ by i.o to $2.0 \mathrm{~m} \mathrm{year}^{-1}$. The data in Section 6 show such changes in thickness. Year-to-year changes in the contribution of basal sliding, which could result not only from thickness changes but also from changes in the extent of "lubrication" that promotes sliding, may also be responsible for the velocity variations.

Velocity measurements over periods of 7 to $24 \mathrm{~d}$ during August suggest that summer velocities may be slightly greater than the yearly velocity at some markers, but no consistent pattern is evident in the observed variations.

\section{Pattern of the velocity field: longitudinal flow component}

Figure 3 displays a pattern of velocities that vary smoothly over the area studied. As shown by the contours in Figure 3, the flow velocity near the center-line remains nearly constant longitudinally over a large region from the $i$ profile down to the $d$ profile. Below the $d$ profile there is a fairly uniform down-stream decrease in flow velocity.

Transverse profiles of flow velocity (Fig. 4) display a flat-bottomed, inverted U-shape of the typical higher-order parabolic form that results from non-linear flow. A detailed profile ( $h$-profile, ${ }^{*}$ Fig. 3 ), with markers I $_{5} \mathrm{~m}$ apart, shows that the velocity gradient close to the margins is steep, but the velocity increases smoothly inward from the margins. Marginal sliding (Section 5) occurs where the margin is a steep bedrock face.

Lack of symmetry about the mid-line of the glacier in some of the velocity profiles (Fig. 4) may be due in part to asymmetry in the bedrock channel. The east wall at profiles $a$ and $b$

* Marker displacements in the $h$ profile were measured only in a direction perpendicular to the profile line, from a single theodolite station. The velocity profile given in Figure 4 was obtained by assuming a flow direction parallel to that measured at $L_{I}$ and $i_{4}$. 

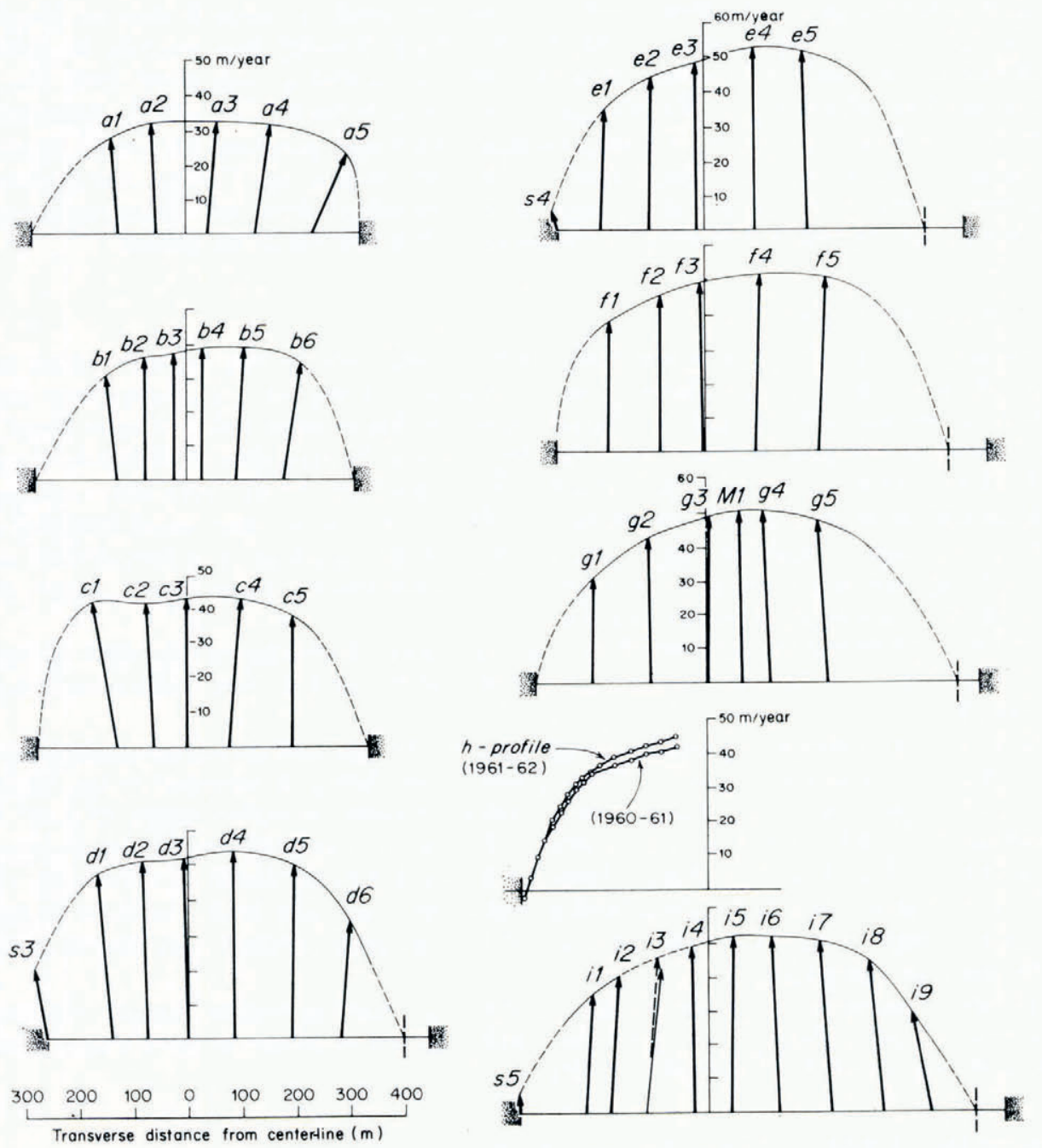

Fig. 4. Transverse profiles of flow velocity. Length of annual motion vectors is shown at a scale enlarged 6.35 times relative to the positions of the velocity markers in the profiles. Flow vectors are for $1957-58$, except at $53-55$. Velocities on $h$ profile represent longitudinal flow component only (see text). "Center-line" corresponds to flow line of stream sheet (Fig. 3 ). The two vectors at i3 are explained in the text (Section 4$)$.

and the west wall at profiles $c, d$, and $e$ are especially steep, and the marginal sliding velocities (Section 5) are high there.

A definite cause of asymmetry in the velocity profile is the prominent bend in the glacier channel between profiles $i$ and $c$ (Fig. 3). The theoretically predicted effect of flow in a horizontally curving channel (W. B. Kamb, unpublished work), is seen clearly in profiles $d$, $e$, and $f$, where the channel curvature is greatest, and also to a certain extent in profiles $g$ and $i$. The effect is a "tilting" of the higher-order parabolic velocity profile toward the inside of the bend, which shifts the maximum velocity toward the outside of the bend (Fig. 4). This occurs in profiles $d, e$, and $f$ despite the suggested asymmetry of the bedrock channel (Fig. I), which would tend to produce the opposite effect. In a separate paper the effect of channel curvature on the flow will be considered in detail. 
The velocity at $i_{3}$ for $1957-58$ (solid arrow in Fig. 4) is anomalously low in relation to the $i$ profile as a whole, and cannot be explained by a local bedrock irregularity as far as known (Fig. I). From $u_{y}$ at $i_{3}$ in $1_{95}^{8-59}$, and from the average change in $u_{y}$ between $1957-5^{8}$ and I 958-59 at $i_{I-2}$ and $i_{4-7}$ (Table I), one would expect $u_{y} \approx 43 . \mathrm{I}$ mear ${ }^{-1}$ at $i_{3}$ in $1957^{-58}$, which corresponds to a velocity $u$ that is reasonable in relation to the $i$ profile as a whole, as shown by the dashed arrow in Figure 4.

\section{Transverse flow component}

In the vicinity of the firn edge (profiles $e, g, i$, Fig. 4) the velocity vectors converge toward the center-line, whereas in the lower part of the ice tongue (profiles $a-d$ ) they diverge from it. Convergence above the firn line and divergence below it might be expected as consequences of accumulation and ablation, from an argument given by Nielsen (1955, p. 8). However, Raymond (I97I, p. 78) shows that the lateral flow expected on this account should take place mainly at depth, and is so observed in Athabasca Glacier. The surface flow convergence at profiles $e, g$, and $i$ occurs below the firn line and hence cannot be due to an accumulation mechanism; it is caused instead by the well-defined narrowing of the channel down-stream between the $i$ and $d$ profiles (Fig. 3). Below the $d$ profile there is a local widening of the channel on the west side, and the outward splaying of velocities at $d_{I}$ and $C_{I}$ is probably in part the result of this. However, the general splaying of the vectors in the lower part of the glacier, which is seen also in the marginal sliding vectors (Section 5), cannot be attributed to channel widening. It can logically be explained as a response to ablation, in spite of its conflict with the observations and reasoning of Raymond (1971, p. 78), by considering the role of areal strain rates (see Section 6 and Kamb and Meier, unpublished).

\section{Plunge of flow vectors}

On the upper profiles the velocity vectors plunge downward from horizontal, whereas on the lowest profiles $(a$ and $b$ ) they are nearly horizontal or directed slightly upward (Fig. 5).
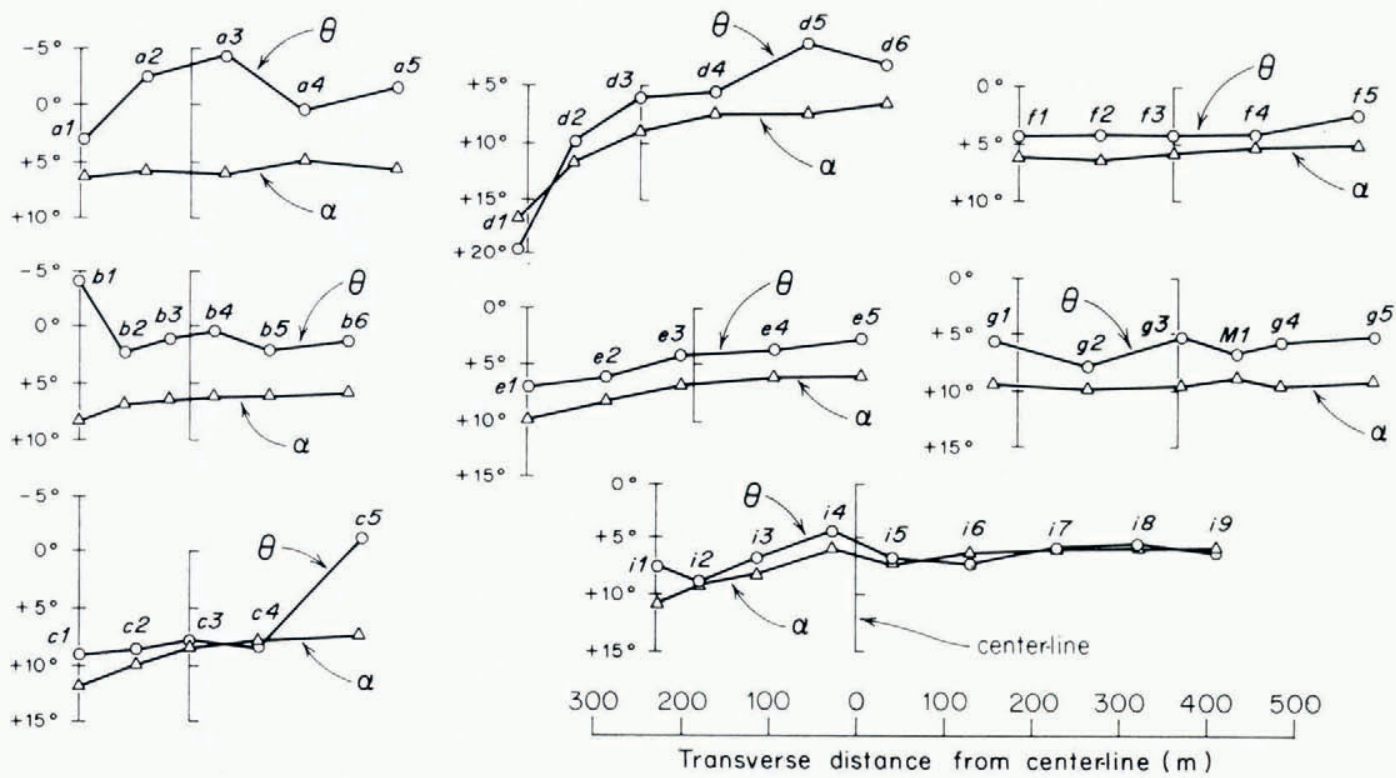

Fig. 5. Plunge of flow vectors $\theta$ in relation to ice surface slope $\alpha$ in transverse profiles. Positive values represent downward plunge in direction of flow. 
Almost everywhere the measured velocity vectors plunge more gently than the local ice surface slope, so that the ice movement is surfaceward, as needed to counterbalance ice loss by ablation. The difference between plunge of the velocity vector $\theta$ and glacier surface slope $\alpha$ is small on the $i$ profile, near the firn line, and increases down-glacier to about $7^{\circ}$ on the $a$ profile, reflecting the progressive increase in annual ice loss down-glacier.

On profiles $d$ and $e, \theta$ and $\alpha$ increase progressively toward the west (Fig. 5). This results from the westward bend in the glacier channel, which requires, geometrically, a westward increase in surface slope. The plunges of the flow vectors increase in response to $\alpha$. In effect, the glacier pours over a small, localized ice fall as it goes around the inside of the bend.

\section{Marginal SLiding}

In August 196r, motion of the ice past the bedrock margin was measured at the seven sites labelled $s_{I-s 7}$ in Figure 3 . At each site the position of a wooden stake fixed in the ice was determined in relation to a stake fixed in the adjacent bedrock by measuring the length, bearing, and plunge of a string stretched between the two stakes. Measurements at successive times give, by difference, the movement vector of the ice. The results are given in Table II.

Table II. Marginal sliding, August ig6r Displacement vector

\begin{tabular}{|c|c|c|c|c|c|c|c|c|}
\hline \multirow[b]{2}{*}{ Site } & \multicolumn{2}{|c|}{ Date } & & \multirow[b]{2}{*}{$\begin{array}{c}\phi \\
\text { deg }\end{array}$} & \multirow[b]{2}{*}{$\begin{array}{c}\theta \\
\operatorname{deg}\end{array}$} \\
\hline & from & to & $\begin{array}{c}\text { Length } \\
\mathrm{m}\end{array}$ & $\begin{array}{c}\text { Horizontal } \\
\mathrm{m}\end{array}$ & $\begin{array}{c}\text { Vertical } \\
\mathrm{m}\end{array}$ & $\begin{array}{c}u \\
\mathrm{~m} / \text { year }\end{array}$ & & \\
\hline \multirow[t]{5}{*}{$S I$} & 3 & I I & $0.24^{\mathrm{I}}$ & 0.216 & +0.102 & 10.0 & 306 & +25 \\
\hline & II * & I9 & 0.234 & 0.216 & +0.090 & 9.9 & 312 & +22 \\
\hline & 19* & 25 & 0.180 & 0.177 & +0.034 & 10.3 & 320 & $+\mathrm{Ir}$ \\
\hline & 25 & $3^{I}$ & o. 192 & o. $19^{2}$ & +0.026 & 11.9 & $3^{17} 7$ & +7 \\
\hline & 3 & $3^{I}$ & 0.835 & o. $79^{6}$ & $+0.25^{2}$ & I 0.4 & $3^{1} 3$ & +17 \\
\hline \multirow[t]{3}{*}{$s 2$} & 3 & I I & 0.219 & 0.219 & -0.028 & $9 \cdot 4$ & 287 & -7 \\
\hline & $19 *$ & 25 & 0.222 & 0.222 & -0.011 & 12.9 & 305 & -3 \\
\hline & $25^{*}$ & $3^{I}$ & $0.20 \mathrm{I}$ & $0.19^{2}$ & -0.059 & 11.9 & 305 & -17 \\
\hline \multirow{4}{*}{$s_{3}$} & 19 & $3^{I}$ & 0.420 & $0.4^{14}$ & -0.070 & 12.6 & 305 & -9 \\
\hline & $\begin{array}{l}\text { I I } \\
\text { I9* }\end{array}$ & I9 & $0.44^{8}$ & $0.44^{8}$ & +0.019 & 20.5 & 333 & +2 \\
\hline & $\begin{array}{l}19^{*} \\
25\end{array}$ & 25 & $0.3^{6} 3$ & 0.363 & -0.009 & 21.4 & $33^{I}$ & $-I$ \\
\hline & $\begin{array}{l}25 \\
\text { I I }\end{array}$ & $3^{I}$ & $0.3^{6} 3$ & $0.3^{6} 3$ & +0.009 & 21.9 & $33^{8}$ & $+I$ \\
\hline \multirow{6}{*}{$s_{4}$} & & $3^{1}$ & I. 172 & 1.1 72 & +0.019 & 21.4 & 334 & $+\mathbf{I}$ \\
\hline & $\begin{array}{l}3 \\
6\end{array}$ & 6 & $0.06 \mathrm{I}$ & 0.058 & -0.016 & $7 \cdot 1$ & 344 & -15 \\
\hline & II * & $\begin{array}{l}\text { I I } \\
\text { I9 }\end{array}$ & 0.085 & 0.082 & -0.013 & $5 \cdot 9$ & $3^{23}$ & -9 \\
\hline & $19^{*}$ & $\begin{array}{l}19 \\
25\end{array}$ & o.1 $5^{8}$ & $0.15^{2}$ & -0.045 & 6.9 & $35^{8}$ & $-\mathrm{I} 6$ \\
\hline & 25 & $\begin{array}{l}25 \\
31\end{array}$ & 0.082 & 0.082 & -0.015 & $4 \cdot 9$ & 335 & $-\mathrm{IO}$ \\
\hline & 3 & $3^{I}$ & $0.13^{1}$ & 0.104 & - 0.079 & 6.2 & $3^{19}$ & -37 \\
\hline \multirow[t]{4}{*}{$s_{5}$} & 12 & & 0.488 & 0.459 & -o.168 & 6.0 & $33^{8}$ & -20 \\
\hline & $19 *$ & $\begin{array}{l}19 \\
25\end{array}$ & $\begin{array}{l}0.15^{2} \\
0.15^{2}\end{array}$ & $\begin{array}{l}0.15^{2} \\
0.140\end{array}$ & 0.000 & 8.o & $\begin{array}{r}17 \\
8\end{array}$ & o \\
\hline & $25^{*}$ & $3 I$ & $\begin{array}{l}0.15^{2} \\
0.137\end{array}$ & $\begin{array}{l}0.140 \\
0.122\end{array}$ & $\begin{array}{l}-0.057 \\
-0.063\end{array}$ & $\begin{array}{l}8.9 \\
7.4\end{array}$ & $\begin{array}{r}8 \\
35^{8}\end{array}$ & $\begin{array}{l}-22 \\
-27\end{array}$ \\
\hline & I 2 & $3^{I}$ & 0.428 & $0.4^{10}$ & -0.120 & $7 \cdot 9$ & 8 & $\begin{array}{l}-1 \\
-\mathrm{I} 6\end{array}$ \\
\hline \multirow[t]{5}{*}{$s 6$} & 6 & I I & 0.104 & O.IOI & -0.026 & 7.2 & I8 & \\
\hline & I I & 19 & 0.168 & o. $15^{8}$ & -0.055 & 7.2 & 8 & $\begin{array}{l}-14 \\
-19\end{array}$ \\
\hline & I9 & 25 & o.1 46 & 0.146 & -0.020 & 9.2 & 3 & -8 \\
\hline & 25 & $3^{1}$ & O. I 16 & O. I Io & -0.042 & 6.7 & 18 & -20 \\
\hline & 6 & $3^{I}$ & $0.53^{1}$ & $0.5^{11}$ & -o.143 & $7 \cdot 5$ & I I & -15 \\
\hline \multirow[t]{4}{*}{$s 7$} & 12 & I9 & o.1 74 & o. 168 & -0.034 & 8.8 & 20 & $-I I$ \\
\hline & 19 & 25 & 0.116 & o. I I 3 & -0.029 & 7.0 & IO & $-\mathrm{I} 4$ \\
\hline & 25 & $3^{I}$ & 0.165 & 0.137 & -0.092 & 8.5 & IO & -34 \\
\hline & 12 & $3^{I}$ & 0.445 & $0.4^{16}$ & -0.155 & 8.0 & 14 & -20 \\
\hline
\end{tabular}

* Marker stake reset. 
Marginal sliding velocities are shown in Figure 3 at a scale enlarged relative to the annual motions of the surface markers, and velocity vectors at s3-5 are shown in Figure 4 at the margin of the nearest velocity profile.

The effect of measurement error on sliding velocities depends on velocity, time interval, string length, and direction. At most stations the velocity vector made a high angle $\left(\approx 65^{\circ}\right)$ with the string direction, and for strings of typical length $\left(0.4^{-1.1} \mathrm{~m}\right)$, the velocity accuracy reflects mainly the accuracy in string direction $\left(0.5^{\circ}\right)$ rather than string length $(0.3 \mathrm{~cm})$. For measurements over time intervals of seven days, the uncertainty is about $0.6 \mathrm{~m} /$ year in $u$ and about $4^{\circ}$ in $\phi$ and $\theta$, for $u \approx 8 \mathrm{~m} /$ year. For the overall measurement period of about $28 \mathrm{~d}$, the uncertainties are a fourth as great, except for errors due to resetting of stakes.

The observed sliding rates (Table II) range from 5 to $22 \mathrm{~m} /$ year. The largest (at $s_{3}$ ) occurs where the glacier is starting over the small ice fall on the inside of the bend (Section 4 ), and the second largest $(s 2)$ is where the glacier descends a steep bedrock slope near the snout. Marginal sliding is $12-47 \%$ of the adjacent center-line surface velocity. Local variations in velocity from site to site are larger than any systematic variation down-glacier. The ratio of marginal sliding to center-line velocity tends to increase down-glacier (as reported for Austerdalsbreen by Glen and Lewis, I96I, p. I I09), mainly because of the down-glacier decrease in center-line velocity. Extrapolation from marginal sliding to basal sliding is not possible, but the data support the probability of large variations in the contribution of basal sliding to total flow velocity from place to place along a valley glacier (Savage and Paterson, I963).

In plan view the marginal slip has a component directed outward from the glacier (Fig. 3), which is consistent with the splaying of the velocity vectors discussed in Section 4 . The divergence from the flow center-line ranges from $18^{\circ}$ to $34^{\circ}$, with $20^{\circ}$ the most representative value.

Inclination of the sliding vector with respect to horizontal $(\theta$ in Table II, plus indicating downward plunge in the direction of motion) is upward except at $s I$ and $s 3$, which are sites where the ice surface slopes steeply down valley.

The consistency in magnitude and direction of movement at sites $55_{-}-7$, which are separated by only a few tens of meters, show that local bedrock configuration on the scale of a few tens of meters does not seriously affect the magnitude and direction of ice movement, contrary to a conclusion of Glen and Lewis (1961, p. I I I 7 ).

The data in Table II suggest temporal variations in marginal sliding. Short-term motions, measured over successive periods of $6-8 \mathrm{~d}$, show variations up to $\mathrm{I}-3 \mathrm{~m} /$ year in rate and up to $\mathrm{IO}^{\circ}-40^{\circ}$ in direction (both $\theta$ and $\phi$ ). In relation to measurement errors, the larger variations in rate appear real, but the variations in direction are questionable, particularly because they increase roughly in inverse proportion to the sliding rates.

The $h$ profile (Figs. 3, 4) provides evidence that where the glacier margin is a steeply sloping moraine, there is no marginal sliding, and the flow velocity decreases continuously to zero at the margin. (The small reversed motion of the outermost $h$ marker nearest the glacier margin (Fig. 4) is probably due to creep of a marginal snowbank.) Along much of the east margin of the glacier the situation appears similar.

\section{Relation between flow and ablation}

The emergence flow component $w_{\mathrm{E}}$ (called emergence velocity $f$ by Meier and Tangborn (r965, p. 56r), "Eisnachschub" by Schimpp (1958, p. 310), and $V_{d}$ by Meier (1960, p. 24)) represents the component of movement that tends to compensate for the net gain or loss of ice from the surface. It is given by

$$
w_{\mathrm{E}}=w+u \tan \alpha
$$

where $\alpha$ is the surface slope. If compensation is not complete, the surface profile changes; 
the rate of change in thickness of the glacier, $\dot{h}$, is related to the annual net balance $a$ and the emergence flow component $w_{\mathrm{E}}$ as follows:

$$
\dot{h}=w_{\mathrm{E}}+a
$$

a represents the net yearly gain $(+)$ or loss $(-)$ of ice at the glacier surface due to accumulation and ablation, measured vertically, in the ice thickness per year.

Values of $w_{\mathrm{E}}$ and $a$, computed at each stake where sufficient data are available, are presented in Table I. The large variation in the results is due only partly to the cumulative effect of several sources of measurement error; actual variations in the flow and the net budget due to local irregularities in topography and albedo are apparently also of importance. A prime source of difficulty is determining the surface slope $\alpha$, which is required for the calculation of $w_{\mathrm{E}}$. Different results are obtained if one uses the local slope measured over distances of 30 to $100 \mathrm{~m}$ in the vicinity of a stake, as in Table I, or averages the slope over longer distances.

In Table III are given the average values of $w_{\mathrm{E}}, a$, and $h$ for each transverse profile during I957-59. The magnitudes of $w_{\mathrm{E}}$ and $a$ both increase down-glacier, but $h$ remains approximately constant, indicating a rate of overall thinning of about $\mathrm{I} \mathrm{m} / \mathrm{year}$. This is consistent with the negative mass budgets measured during this time by LaChapelle (1959, p. 445; i96o, p. i9).

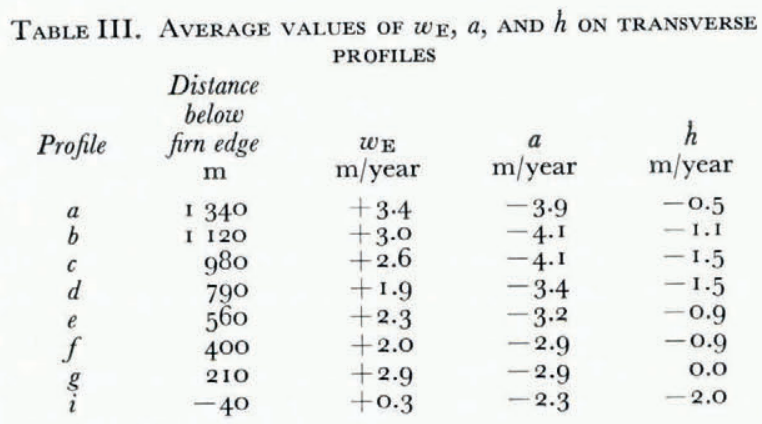

\section{Surface strain-Rate distribution}

\section{Measurement method and accuracy}

Surface strain-rates are calculated from the velocity measurements by modification of a procedure previously employed (Meier, $196 \%$, p. 32). In principle the instantaneous surface strain-rates are given by

$$
\dot{e}_{x x}=\partial u_{x} / \partial x, \quad \dot{e}_{y y}=\partial u_{y} / \partial y, \quad \dot{e}_{x y}=\frac{1}{2}\left(\partial u_{x} / \partial y+\partial u_{y} / \partial x\right)
$$

where $u_{x}$ and $u_{y}$ are the instantaneous velocity components, and where the $x$ - and $y$-axes here are tangent to the local ice surface. The velocity gradients were determined from a weighted average of velocity differences read across a square grid $100 \mathrm{~m}$ on the side on contoured plots of $u_{x}$ and $u_{y}$. Since $u_{x}$ and $u_{y}$ are measured over a finite period of time, the calculated strain-rates are spatial and temporal averages. The known time- and spacevariations in the velocities are sufficiently small that the difference between the average strain-rates and instantaneous local values is negligible in comparison with the measurement errors. Use of the horizontal $x, y$ coordinate system defined in Section 3 gives negligible errors because the surface slopes are small.

Principal strain-rates $\dot{\epsilon}_{1}$ (greatest) and $\dot{\epsilon}_{2}$ (least), and the angle $\psi$ between the $\dot{\epsilon}_{1}$ axis and the $x$-axis (positive from $+x$ toward $+y$ ) were determined from the standard formulae

$$
\dot{\epsilon}_{1}, \dot{\epsilon}_{2}=\frac{1}{2}\left(\dot{e}_{x x}+\dot{e}_{y y}\right) \pm\left[\frac{1}{4}\left(\dot{e}_{x x}-\dot{e}_{y y}\right)^{2}+\dot{e}_{x y^{2}}\right]^{\frac{1}{2}}, \quad \tan 2 \psi=\frac{2 \dot{e}_{x y}}{\dot{e}_{x x}-\dot{e}_{y y}} .
$$


The results are given in Table IV and Figure 6.

Table IV. Surface Strain-Rates, $1957-58$

\begin{tabular}{|c|c|c|c|c|c|c|c|}
\hline $\begin{array}{c}x \\
\mathrm{~m}\end{array}$ & $\begin{array}{l}y \\
\mathrm{~m}\end{array}$ & $\begin{array}{c}\dot{e}_{x x} \\
\text { year }^{-1}\end{array}$ & $\begin{array}{c}\dot{e}_{y y} \\
\text { year }^{-\mathrm{I}}\end{array}$ & $\begin{array}{c}\dot{e}_{x y} \\
\text { year }^{-1}\end{array}$ & $\begin{array}{c}\dot{\epsilon}_{1} \\
\text { year }^{-1}\end{array}$ & $\begin{array}{c}\dot{\epsilon}_{2} \\
\text { year }^{-1}\end{array}$ & $\begin{array}{c}\psi \\
\text { deg }\end{array}$ \\
\hline $35^{\circ}$ & I $95^{\circ}$ & -0.012 & +0.004 & +0.024 & +0.021 & -0.029 & 54 \\
\hline $45^{\circ}$ & I $95^{\circ}$ & +0.007 & -0.013 & +0.032 & +0.031 & -0.037 & 37 \\
\hline $55^{\circ}$ & I $95^{\circ}$ & +0.021 & -0.027 & +0.031 & $+0.03^{6}$ & -0.042 & 26 \\
\hline $35^{\circ}$ & I 850 & -0.005 & +0.023 & +0.020 & +0.033 & -0.015 & 62 \\
\hline $45^{\circ}$ & I 850 & -0.001 & +0.009 & +0.027 & $+0.03 \mathrm{I}$ & -0.023 & 50 \\
\hline $55^{\circ}$ & I 850 & +0.007 & -0.016 & +0.024 & +0.022 & -0.031 & $3^{2}$ \\
\hline $65^{\circ}$ & I 850 & +o.019 & $-0.03^{8}$ & -0.003 & +0.019 & -0.038 & -3 \\
\hline $45^{\circ}$ & I $75^{\circ}$ & -0.016 & +0.035 & +0.030 & +0.049 & -0.030 & 65 \\
\hline $55^{\circ}$ & $175^{\circ}$ & +0.009 & -0.002 & +0.029 & +0.033 & -0.026 & 39 \\
\hline 650 & I $75^{\circ}$ & +0.025 & -0.054 & +0.015 & +0.028 & -0.057 & IO \\
\hline $75^{\circ}$ & I $75^{\circ}$ & +0.012 & $-0.09 \mathrm{I}$ & -0.013 & +0.013 & -0.093 & -7 \\
\hline $55^{\circ}$ & I 650 & +0.086 & -0.052 & +0.075 & +0.119 & -0.085 & 24 \\
\hline 650 & I $65^{\circ}$ & +0.022 & -0.058 & +0.028 & +0.031 & -0.067 & 17 \\
\hline $75^{\circ}$ & I 650 & +0.014 & -0.067 & +0.001 & +0.014 & -0.033 & I \\
\hline $85^{\circ}$ & I 650 & +0.024 & -0.083 & +0.042 & +0.038 & -0.098 & -19 \\
\hline $55^{\circ}$ & I $55^{\circ}$ & +0.079 & -0.028 & +0.042 & +0.094 & -0.042 & 19 \\
\hline 650 & I $55^{\circ}$ & +0.023 & $-0.03^{8}$ & +0.006 & +0.024 & -0.039 & 6 \\
\hline $75^{\circ}$ & I $55^{\circ}$ & +0.026 & -0.011 & -0.014 & +0.031 & -0.016 & -19 \\
\hline 850 & I $55^{\circ}$ & +0.022 & -0.027 & $-0.03^{2}$ & +0.038 & -0.043 & -26 \\
\hline 650 & I $45^{\circ}$ & +0.015 & +0.026 & -0.011 & +0.033 & +0.008 & -59 \\
\hline $75^{\circ}$ & I $45^{\circ}$ & +0.015 & +0.018 & -0.009 & +0.026 & +0.007 & $-5^{\circ}$ \\
\hline 850 & I $45^{\circ}$ & +0.008 & -0.013 & -0.018 & +0.018 & -0.023 & -30 \\
\hline 650 & I $35^{\circ}$ & -0.004 & +0.009 & 0.000 & $+0.02 \mathrm{I}$ & -0.007 & -70 \\
\hline $75^{\circ}$ & I $35^{\circ}$ & -0.003 & 0.000 & -0.011 & +0.010 & -0.013 & -49 \\
\hline 850 & I $35^{\circ}$ & -0.004 & -0.007 & $-0.02 \mathrm{I}$ & +0.015 & -0.027 & -43 \\
\hline 650 & I $25^{\circ}$ & -0.017 & -0.003 & +0.013 & +0.005 & -0.025 & 59 \\
\hline $75^{\circ}$ & $125^{\circ}$ & -0.006 & -0.006 & -0.009 & +0.003 & -0.015 & -45 \\
\hline $85^{\circ}$ & I $25^{\circ}$ & -0.002 & +0.002 & -0.016 & +0.016 & -0.016 & -49 \\
\hline 650 & 1150 & -0.008 & +0.004 & +0.023 & +0.021 & -0.026 & 57 \\
\hline $75^{\circ}$ & I $53^{\circ}$ & -0.005 & +0.004 & -0.002 & +0.004 & -0.005 & -78 \\
\hline 850 & I $53^{\circ}$ & -0.008 & +0.005 & -0.017 & +0.017 & -0.020 & -56 \\
\hline $55^{\circ}$ & 1050 & -0.005 & -0.021 & +0.034 & +0.022 & -0.048 & $3^{8}$ \\
\hline 650 & 1050 & -0.001 & 0.000 & +0.015 & +0.015 & -0.016 & 46 \\
\hline $75^{\circ}$ & 1050 & -0.006 & +0.004 & -0.002 & +0.004 & -0.006 & $-8 I$ \\
\hline $8 j o$ & I 050 & -0.011 & +0.008 & -0.012 & +0.014 & -0.017 & -64 \\
\hline $55^{\circ}$ & $95^{\circ}$ & 0.000 & -0.042 & $+0.03^{1}$ & +0.017 & -0.059 & 28 \\
\hline $65^{\circ}$ & $95^{\circ}$ & -0.016 & -0.009 & +0.020 & -0.004 & -0.030 & 44 \\
\hline $75^{\circ}$ & $95^{\circ}$ & -0.013 & -0.001 & +0.001 & -0.001 & -0.013 & 85 \\
\hline $85^{\circ}$ & $95^{\circ}$ & -0.018 & +0.002 & -0.012 & + 0.008 & -0.024 & -65 \\
\hline $55^{\circ}$ & 850 & +0.002 & -0.049 & +0.008 & +0.003 & -0.050 & 9 \\
\hline 650 & 850 & -0.020 & -0.022 & -0.010 & -0.011 & $-0.03^{1}$ & -48 \\
\hline $75^{\circ}$ & 850 & -0.018 & -0.007 & -0.007 & 0.000 & -0.025 & -64 \\
\hline 850 & 850 & -0.018 & +0.004 & -0.013 & +0.010 & -0.024 & -65 \\
\hline $75^{\circ}$ & $75^{\circ}$ & -0.047 & -0.007 & -0.018 & 0.000 & -0.054 & -69 \\
\hline 850 & $75^{\circ}$ & -0.026 & +0.004 & -0.018 & +0.012 & -0.034 & -65 \\
\hline
\end{tabular}

The largest source of error in the strain-rates is due to the subjective procedure of constructing the velocity contour maps from the locally measured velocity values. Discrepancies among the results of this procedure as carried out by different people indicate an uncertainty of order \pm o.or year ${ }^{-1}$ from this cause, rather larger than the uncertainty of about \pm 0.005 year $^{-1}$ expected from the velocity measurement errors. Uncertainty in the strain-rates is largest near the periphery of the marker field, where control for the velocity contours is sparsest. *

* The anomalously low $u_{y}$ measured at i3 for 1957-58 (Section 4) causes large anomalies in the calculated strain-rate field in its vicinity. The strain-rate data in Table IV and Figure 6 are based on the more reasonable $u_{y}$ value $4^{1} \cdot 3 \mathrm{~m}$ year $^{-1}$ (see Section 4 ). 


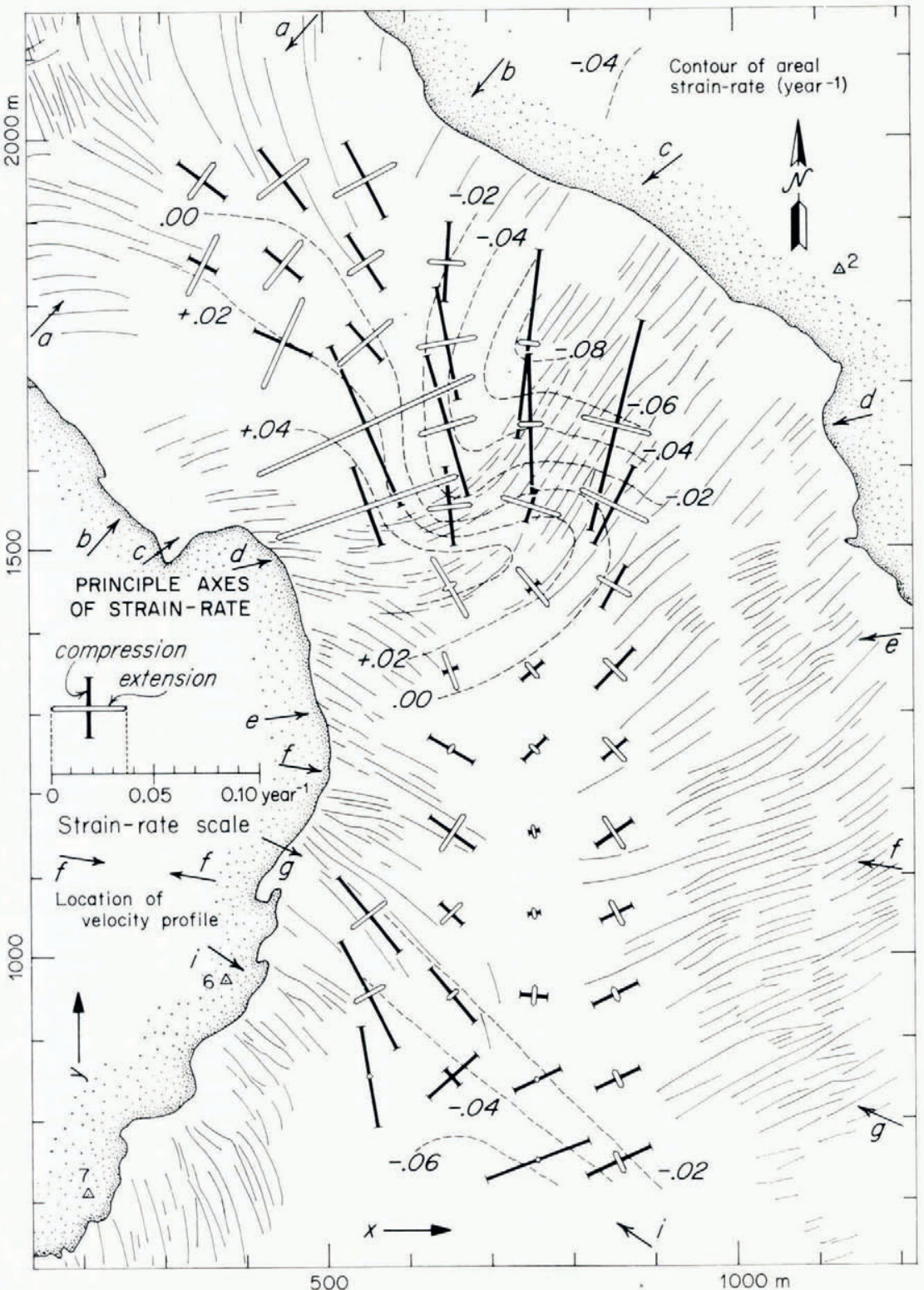

Fig. 6. Surface strain-rates, shown in terms of the orientation and magnitude of the principal strain-rates. Dashed contours show the areal strain-rate $\dot{\epsilon}_{1}+\dot{\epsilon}_{2}$, in units of year ${ }^{-1}$. Thin lines are freshly formed crevasses.

\section{Strain-rate pattern}

The pattern of strain-rates (Fig. 6) is in some respects similar to that on Saskatchewan Glacier (Meier, 1960, p. 33), but it is more complicated because of complexities in channel geometry. The Saskatchewan pattern is dominated by marginal shear, in which the principal axes tend toward an orientation at $45^{\circ}$ to the flow direction. This effect is visible in most peripheral parts of the strain-rate field in Figure 6, but velocity control does not extend 
fully into the areas of greatest marginal strain. The largest marginal shear strain-rates shown in Figure 6 , uncomplicated by other effects, are $\dot{\epsilon}_{1}-\dot{\epsilon}_{2} \approx 0.08$ year ${ }^{-1}$, much smaller than the maximum of about 0.3 year $^{-1}$ found on Saskatchewan Glacier. Shear strain-rates of 0.19 year ${ }^{-1}$ over the intervals $i 8-i g$ and $d_{5}-d 6$ are indicated by the measured velocities (in ${ }^{-95^{8}}-59$; 0.17 year $^{-1}$ in $1957^{-58}$ ), but these high strain-rates do not appear in Figure 6 because the velocity control is not dense enough to permit reliable velocity contours to be drawn in these parts of the map. On the $h$ profile the marginal shear strain-rate indicated by the velocity data in Figure 4 is $0.4^{8}$ year $^{-1}$.

The largest non-marginal strain-rates observed on Blue Glacier are in the reach between the $c$ and $d$ profiles. Near the $d$ profile, longitudinal extension of about 0.03 year $^{-1}$ is visible in Figure 6 where marginal shear does not dominate, for example at $(x, y)=(650 \mathrm{~m}, \mathrm{I} 450 \mathrm{~m})$. Near the $c$ profile there is pronounced longitudinal compression, reaching rates of 0.08 year $^{-1}$. These effects relate directly to a localized steepening of the glacier surface profile: the average surface slope at the flow center-line over the $200 \mathrm{~m}$ interval between the $c$ and $d$ profiles is $9.1^{\circ}$, whereas it is $6.3^{\circ}$ over the interval just below and $7.1^{\circ}$ over the interval just above.

Below the $c$ profile, longitudinal compression at a rate of about 0.03 year ${ }^{-1}$ reflects the longitudinal velocity decrease in this area, and transverse extension at about the same rate reflects the splaying of the velocity vectors (Section 4). In the interval between the $d$ and $g$ profiles, longitudinal strains are small and the pattern is dominated by marginal shear. Up-stream from this, prominent compressions, more or less longitudinal, are related to higher surface slopes in the area farther up-stream mostly outside the region of velocity control.

Over much of Figure 6 the "stress center-line" of the pattern, where one of the principal axes of strain-rate aligns with the flow direction, is displaced westward from the geometrical center-line of the channel. This results from the westward-curving flow (Fig. 3). The prominent effect of curving flow on the strain-rate pattern is a novel feature of the strain-rate results for Blue Glacier; apparently the effect has not been noticed previously in studies of other glaciers, although channel curvature is a common feature. In the vicinity of profiles $e, f$, and $g$, the curvature is almost constant and the flow is almost perfectly circular, that is, the velocity vectors are essentially perpendicular to a radius vector originating from a point fixed in space. In flow around a bend of constant curvature, the surface velocity and strain-rate fields are best described in polar coordinates $r, \phi$ with origin at the center of curvature. If the stream lines form circles about this center, then the velocity field is completely described by the $\phi$-directed velocity component $v_{\phi}(r, \phi)$. When $v_{\phi}$ depends only on $r$, as is approximately true for profiles $e-g$, the only non-vanishing surface strain-rate component is the shear strainrate $\dot{e}_{r_{\phi}}$, which is given by

$$
\dot{e}_{r_{\phi}}=\frac{1}{2}\left(\frac{\partial v_{\phi}}{\partial r}-\frac{v_{\phi}}{r}\right) \text {. }
$$

The term $-v_{\phi} / r$ affects the shear-strain-rate distribution in a curving channel but not in a straight one $(r \rightarrow \infty)$. It shifts the "stress center-line", where $\dot{e}_{r_{\phi}}=0$, away from the "flow center-line" (where $v_{\phi}$ is a maximum) and toward the inside of the bend. Figure 7 shows that the channel curvature near profile $f$ in Blue Glacier should shift the stress center-line about I $40 \mathrm{~m}$ toward the inside of the bend. The solid curve labelled $\dot{e}_{r_{\phi}}$ in Figure 7 is calculated from Equation (2) on the basis of the velocity profile $v_{\phi}(r)$ shown, which approximates the observed profiles $e-g$ (Fig. 4). The center of curvature is about I $400 \mathrm{~m}$ from the flow centerline. For comparison, the dashed curve in Figure 7 shows the corresponding shear-strain-rate distribution for a straight channel.

The plotted points in Figure 7 are observed shear strain-rates $\pm \frac{1}{2}\left(\dot{\epsilon}_{1}-\dot{\epsilon}_{2}\right)$ obtained from the data in Table IV for points in the vicinity of profiles $e-g$, with choice of sign dictated by the orientation of the local principal strain-rate axes relative to the local flow direction (Fig. 6). The quantity $\pm \frac{1}{2}\left(\dot{\epsilon}_{1}-\dot{\epsilon}_{2}\right)$ represents $\dot{e}_{r_{\phi}}$ if the principal axes are at $45^{\circ}$ to the flow direction 
(absence of longitudinal or transverse strains), which is generally true in the interval $e-g$. The good correspondence between observed and calculated strain-rates verifies the effect of the curvature term $-v_{\phi} / r$ on the strain-rate distribution.

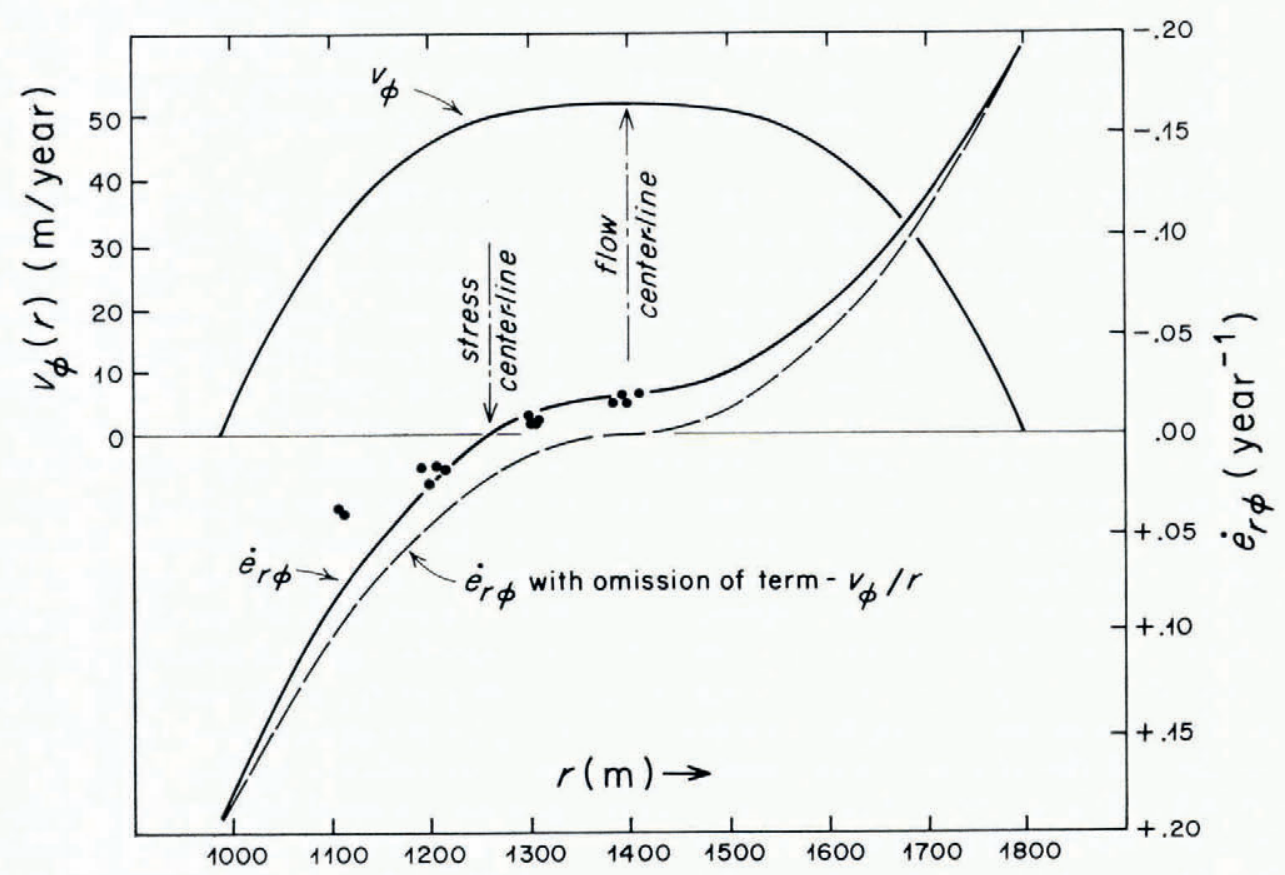

Fig. 7. Effect of flow curvature on distribution of shear strain-rate $\dot{e}_{r_{\phi}}$ in a transverse profile. Effect of curvature term - $v_{\phi} / r$ is to displace stress center-line (where $\dot{e}_{r_{\phi}}=o$ ) away from flow center-line as shown. Plotted points are observed shear strain-rate values for $y=950-1250 \mathrm{~m}$, as explained in text.

\section{Relation of crevasse pattern to strain-rate distribution}

Crevasses form when the greatest principal extending strain-rate exceeds about 0.03 year $^{-1}$. The crevasse orientation tends to be perpendicular to the principal extension axis, as shown in Figure 6, in which crevasse orientations have been plotted from vertical aerial photographs (see Allen and others, r960, plate ${ }_{1}$ B). The orientations plotted are those of the thinnest, most recently formed crevasses.

Longitudinal extension superimposed upon marginal shear in the region near profile $d$ causes the formation of curving transverse crevasses there. Toward the snout, longitudinal compression gives rise to a typical splaying crevasse pattern, which is prominent from profile $a$ downstream. The two types of crevasse pattern correlate closely with the strain-rate field in the way expected by Nye (1952).

The effect of channel curvature on the surface strain-rate distribution is reflected in asymmetry of the marginal crevasse fields: on the outside of the bend (east side of Blue Glacier) the crevasse field is much wider than on the inside (Fig. 6). This asymmetry is direct evidence that the stress center-line is shifted from the channel center-line toward the inside of the bend. The effect is most pronounced near profile $d$, where the flow curvature is greatest. The stress center-line is here made visible by the curving transverse crevasses: it is located where the crevasse orientation is perpendicular to the flow direction. Near $(x, y)=(650 \mathrm{~m}$, I $450 \mathrm{~m}$ ) the stress center-line is displaced half-way from the channel center toward the inside (west) margin. 


\section{Areal strain-rate}

The distribution of $\dot{e}_{x x}+\dot{e}_{y y}$ over the glacier surface is shown by contours in Figure 6 , based on the data in Table IV. At the southern edge of the area studied, there is a strong southward increase in compressive areal strain-rate, which is doubtless related to the smaller ice thickness and greater surface slope to the south (Figs. I, 2). Within the area studied, $\dot{e}_{x x}+\dot{e}_{y y}$ is for the most part small (magnitude $<0.02$ year $^{-1}$ ), except in the vicinity of profiles $c$ and $d$, where there is a pronounced lateral gradient from -0.08 year ${ }^{-1}$ on the east side to +0.05 year $^{-1}$ on the west side. The $S$ shape of the contours defining this lateral gradient indicates superposition of a band of somewhat higher areal expansion rate $\left(\approx+0.03\right.$ year $\left.^{-1}\right)$ extending across the glacier just south of the $d$ profile, and a similar band of relatively high areal compression rate $\left(\approx-0.03\right.$ year $\left.^{-1}\right)$ just south of the $c$ profile. The position of the extensional band correlates with the down-glacier increase in surface slope mentioned previously and with transverse crevassing (Fig. 6), while the compressional band similarly correlates with the subsequent down-glacier decrease in surface slope and termination of the transverse crevassing. The large lateral gradient in areal strain-rate near profiles $c$ and $d$ does not have a certain explanation but may reflect a longitudinal variation in the shape of the glacier crcsssection, such that the glacier becomes relatively thicker down-stream on the north-east side and relatively thinner on the south-west side. Figure 7 suggests that above the $d$ profile, the channel is somewhat asymmetric with the deepest point lying toward the west side, whereas below the $c$ profile the asymmetry is reversed.

The relation between areal strain-rate and net balance is discussed in a separate paper (Kamb and Meier, unpublished).

\section{Ice discharge}

Continuity requires that any change in ice discharge down-stream must appear as a nonzero emergence flow $w_{\mathrm{E}}$ at the glacier surface. Integration of measured $w_{\mathrm{E}}$ values gives discharge variations that should be compatible with discharge values obtained from measured flow velocities and channel cross-sectional areas. The degree of agreement between discharges calculated in these two independent ways is a measure of the extent to which the available velocity data and flow theory give reliable information on the flow of the glacier as a whole. Related types of calculation, on a somewhat different basis, have been carried out earlier by Meier (1960, p. 45) for Saskatchewan Glacier and Meier and Tangborn (1965, p. 562) for South Cascade Glacier.

\section{The stream sheet}

Because flow velocity and channel shape are more reliably known in Blue Glacier near the glacier center than near the margins, the discharge calculation is carried out along a thin "stream sheet" that follows a flow line near the center. The stream sheet consists of an ice volume bounded laterally by two surfaces parallel to flow lines a small distance apart, and bounded top and bottom by the glacier surface and bed. The flow line is shown in horizontal projection in Figure 3. Horizontal distance measured along the projected flow line is designated by $\xi$, and is measured from the origin marked "o" near $S_{2}$ and $i_{4}$ in Figure 3 . The projected flow line is drawn parallel to the horizontal component $u$ of the velocity vector at the glacier surface. The velocity vectors at depth are assumed parallel in horizontal projection to those at the glacier surface vertically above, hence the lateral boundaries of the stream sheet are taken as a pair of vertical surfaces, the thickness of the sheet depending only on $\xi$. This assumption is more likely to be satisfied near the center-line of the glacier than elsewhere: it is a definite limitation on the validity of the flux calculations. 
The width $\zeta_{\mathrm{s}}$ of the stream sheet is taken arbitrarily as $\zeta_{\mathrm{s}}=\mathrm{I} .00 \mathrm{~m}$ at $\xi=0$, and elsewhere it is computed by integration of

$$
\frac{d \zeta_{\mathrm{s}}}{d \xi}=\frac{\zeta_{\mathrm{s}} \dot{e}_{\zeta \zeta}}{u_{\mathrm{s}}}
$$

where $u_{\mathrm{S}}$ is the horizontal component of the surface velocity, and $\dot{e}_{\zeta \zeta}$ is the extension rate transverse to the flow line. Values of $\dot{e}_{\zeta \zeta}$ are obtained from the strain-rate data (Table IV) by a local rotation of coordinate axes through the angle $\phi$ between the projected flow line and the $v$-axis. Computed $\zeta_{\mathrm{s}}$ values are given in Table V.

\section{Table V. Continuity and flux Calculations}

\begin{tabular}{|c|c|c|c|c|c|c|c|c|c|c|}
\hline Marker & $\begin{array}{c}\xi \\
\mathrm{m}\end{array}$ & $\begin{array}{l}\zeta_{\mathrm{s}} \\
\mathrm{m}\end{array}$ & $\begin{array}{c}u_{\mathrm{s}} \\
\mathrm{m} \mathrm{year}^{-1}\end{array}$ & $\begin{array}{c}h \\
\mathrm{~m}\end{array}$ & $\sin \alpha$ & $\begin{array}{c}Q_{\mathrm{C}} \\
\mathrm{m}^{3} \text { year }^{-1} \\
\times \mathrm{I}^{3}\end{array}$ & $\begin{array}{c}Q_{\mathrm{F}} \\
\mathrm{m}^{3} \text { year }^{-1} \\
\times \mathrm{IO}^{3}\end{array}$ & $\begin{array}{c}u_{\mathrm{B}} \\
\text { m year }\end{array}$ & $\sin \alpha^{\star}$ & $\begin{array}{c}u_{\mathrm{B}}{ }^{\star} \\
\text { m year }\end{array}$ \\
\hline$i_{5}$ & -115 & 1.05 & $5^{2.2}$ & 230 & 0.13 & 10.5 & $9 \cdot 5$ & $-I I$ & 0.115 & 10 \\
\hline$i_{4}, S_{2}$ & 0 & I.OO & 49.6 & 254 & O. IO & 10.4 & 10.4 & 7 & 0.10 & 7 \\
\hline$g_{3}$ & 160 & 0.97 & 49.0 & 255 & 0.08 & 10.0 & 11.0 & 26 & 0.10 & 7 \\
\hline$f_{3}$ & 300 & 0.93 & 49.2 & $(251)$ & 0.095 & $9 \cdot 7$ & 10.6 & I 2 & 0.095 & I 1 \\
\hline e3 & 495 & $0.9^{2}$ & $4^{8.5}$ & 235 & 0.12 & $9 \cdot 3$ & 8. I & -6 & 0.095 & 21 \\
\hline$d_{3}$ & 715 & 0.93 & $5^{1} \cdot 5$ & (203) & O. I 55 & 8.7 & $7 \cdot 3$ & $-\mathrm{I} 2$ & O.I I & 26 \\
\hline$c_{3}$ & 910 & 0.97 & 43.4 & 208 & 0.14 & 8.1 & 6.6 & -9 & 0.09 & 23 \\
\hline$b_{3} / 4$ & 1050 & 1.08 & 38.5 & (202) & 0.115 & 7.6 & $7 \cdot 3$ & IO & 0.10 & 19 \\
\hline$a / b$ & I 185 & 1.18 & 35.0 & 184 & 0.10 & 7.1 & 7.1 & 21 & 0.10 & $2 \mathrm{I}$ \\
\hline$a_{3}$ & I 275 & 1.24 & 32.8 & (I 74) & 0.095 & 6.7 & 6.7 & 25 & 0.095 & 25 \\
\hline
\end{tabular}

There is a general agreement between the observed width of ice stream $B_{I}$ (Fig. 3; see Allen and others, I96o, p. 607 and fig. 3) and the calculated $\zeta_{\mathrm{s}}$ values scaled to match $\mathrm{B}_{\mathrm{I}}$ at $\xi=\mathrm{o}$ (Fig. 8). This provides a rough check on the calculation of $\zeta_{\mathrm{s}}(\xi)$.

\section{Continuity calculation of ice flux $Q_{\mathrm{C}}$}

For a stream sheet of infinitesimal width $\zeta_{\mathrm{s}}$, the vertically integrated form of the continuity condition is

$$
\frac{\partial Q_{\mathrm{C}}}{\partial \xi}=\zeta_{\mathrm{s}}\left(-w_{\mathrm{S}}+u_{\mathrm{s}} \frac{\partial z_{\mathrm{s}}}{\partial \xi}\right)=\zeta_{\mathrm{s}} w_{\mathrm{E}}
$$

where the subscript $\mathrm{S}$ refers to surface values along the stream sheet. Flux variation along the flow line is calculated by integrating Equation (4) in the form

$$
d Q_{\mathbf{C}}=\zeta_{\mathbf{s}}\left(-w_{\mathbf{S}} d \xi+u_{\mathbf{S}} d z_{\mathbf{S}}\right)
$$

which uses the directly measured velocity data and avoids the intermediate quantity $w_{\mathrm{E}}$. In Blue Glacier, the dominating term in Equation (5) is $\zeta_{\mathrm{s}} u_{\mathrm{S}} d z_{\mathrm{S}}$. Smooth curves used in the integration of Equation (5) are shown and compared with measured data points in Figure 8. The $u_{\mathrm{S}}(\xi)$ curve is based on the velocity data for 1957-58. Observed $w$ values for both $1957^{-5}$ and $195^{8-59}$ are used in drawing the $w(\xi)$ curve, on the grounds that the annual variation, if percentage-wise the same as for $u$, would be small compared to the scatter in measured $w$ values. Measured ablation values $a$ are compared in Figure 8 with the curve $-w_{\mathrm{E}}(\xi)$, computed on the basis of the surface slope $\alpha$ averaged over roo $\mathrm{m}$ intervals $\Delta \xi$. The ablation values follow the $-w_{\mathbf{E}}(\xi)$ curve systematically, with small discrepancies indicating ice thinning of $\mathrm{o}$ to $\mathrm{I} \mathrm{m}_{\text {year }}^{-1}$, according to Equation ( $\mathrm{I}$ ). However, anomalously large discrepancies occur in the interval $\xi=800$ to $\mathrm{I}$ ooo $\mathrm{m}$, where $-w_{\mathrm{E}}(\xi)$ has a sharp, localized minimum. This is caused by the unusually large negative values of $w$ measured on the $c$ profile for $1957-58$, which contrast sharply with those on the same profile for $195^{8-59}$ 


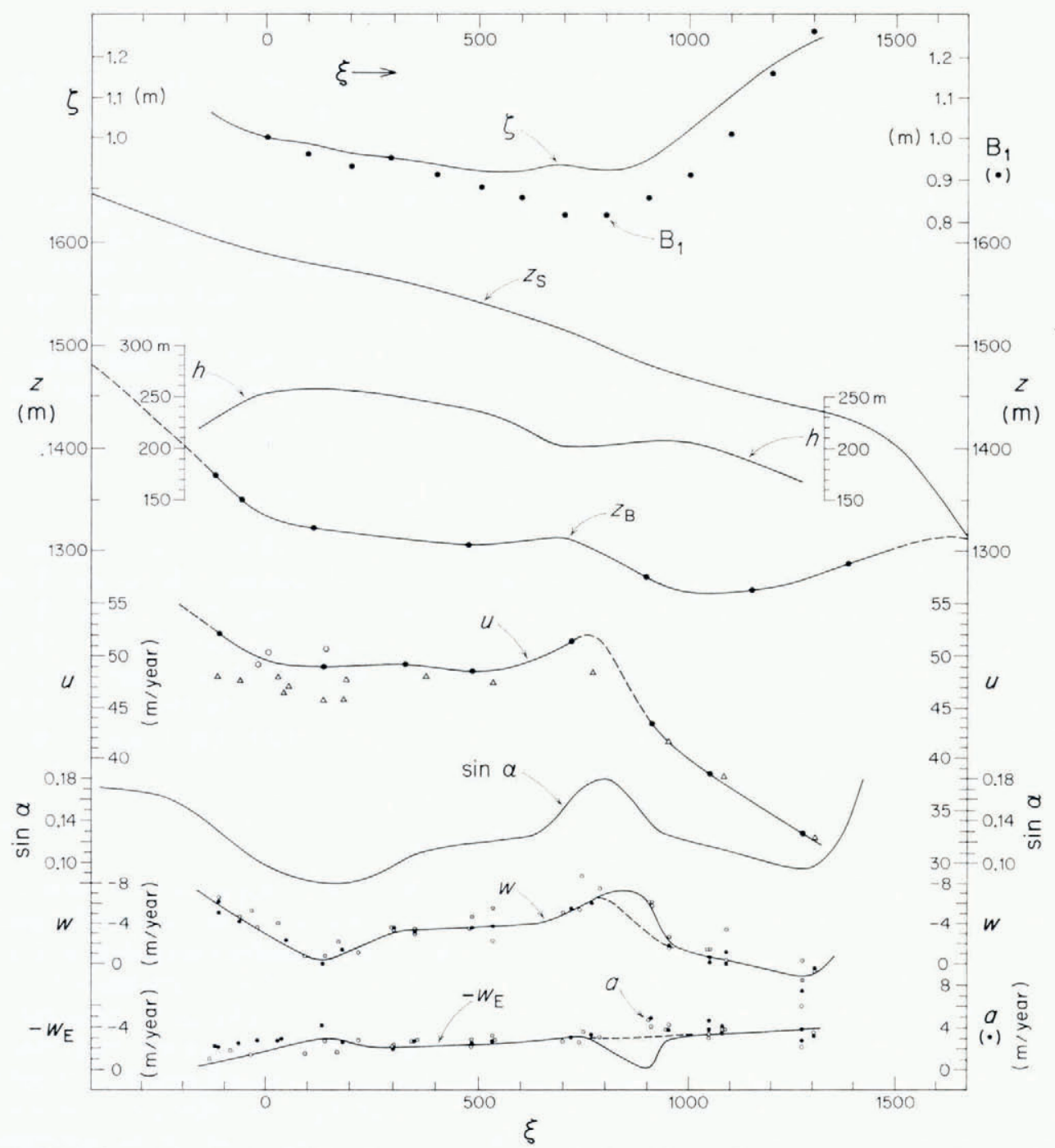

Fig. 8. Variables entering into the ice flux calculations plotted as a function of longitudinal coordinate $\xi$ along the stream sheet. Points are measured data; smoothed curves are used in calculations. Open point symbols represent measurement points dislant from the stream sheet. $\zeta$ is the width of the stream sheet, and points marked " $B_{\mathrm{I}}$ " represent the width of ice stream $B_{\mathrm{I}}$ (Fig. 2), scaled to match $\zeta$ at $\xi=o . z_{\mathrm{S}}$ and $z_{\mathrm{B}}$ are ice surface and bottom elevations, $h$ is ice thickness, $u$ is center-line horizontal surface velocity, $\alpha$ is surface slope averaged over $100 \mathrm{~m}$ intervals $\Delta \xi, w$ is vertical component of velocity, w $w_{\mathrm{E}}$ is emergence flow component, and $a$ is annual ice balance (shown as measured points).

(Table I). Measured $w$ values in general correlate well with the curve of surface slope $\alpha(\xi)$ (Fig. 8), but for the $c$ profile in $1957-5^{8}$, the correlation is strongly violated. We choose to remove this unexplained anomaly by disregarding the $c$-profile $w$ values for $1957-58$ and using the dashed curve $w(\xi)$ in the interval $\xi=800$ to I ooo $\mathrm{m}$, which correlates with $\alpha(\xi)$ (Fig. 8). This gives fluxes down-stream from $c$ that are lower by $230 \mathrm{~m}^{3}$ year-1 than would be obtained if the $c$-profile $w$ values were not disregarded (solid curve $w(\xi)$ between $\xi=800$ and $\mathrm{I}$ ooo $\mathrm{m}$ in Figure 8).

Flux values $Q_{\mathrm{c}}(\xi)$ obtained from integration of Equation (5) in steps $\Delta \xi=100 \mathrm{~m}$, starting from $Q_{\mathrm{C}}(\mathrm{o})=10.4 \times 1 \mathrm{O}^{3} \mathrm{~m}^{3}$ year $^{-1}$ (see below), are given in Table $\mathrm{V}$. 
Flux calculation from flow at depth $\left(Q_{\mathbf{F}}\right)$

Bore hole $S_{2}$ near $\xi=0$ provides flow velocity data to a depth of $180 \mathrm{~m}$ (Shreve and Sharp, 1970). The velocity profile $u(z)$ fits the empirical formula

$$
u_{\mathrm{S}}-u(z)=C\left(z_{\mathrm{S}}-z\right)^{n+1}
$$

where $C=\mathrm{I} .80 \times \mathrm{IO}^{-8} \mathrm{~m}^{-n}$ year $^{-1}$ and $n=2.9$. Because this corresponds tolerably with laboratory evidence on the flow law (Glen, 1955) and with some measurements on other glaciers (Mathews, r 959), * it is reasonable to extrapolate Equation (6) from depth $180 \mathrm{~m}$ to the bottom at $254 \mathrm{~m}$. A basal sliding velocity $u_{\mathrm{B}}=7 \mathrm{~m}$ year ${ }^{-1}$ is indicated for $1957-58$. By integration of Equation (6), the flux $Q_{\mathrm{F}}(\mathrm{o})=\mathrm{I} 0.4 \times 10^{3} \mathrm{~m}^{3}$ year ${ }^{-1}$ is obtained for $1957-5^{8}$.

If Equation (6) represents a flow law of general validity, in which the shear strain-rate depends non-linearly on shear stress, and in which shear stress is proportional to depth and to $\sin \alpha$, then the ice flux in the stream sheet should be given everywhere by

$$
Q_{\mathrm{F}}=\left[u_{\mathrm{S}} h-\Delta q_{0}\left(\frac{\sin }{\sin } \frac{\alpha}{\alpha_{0}}\right)^{n}\left(\frac{h}{h_{0}}\right)^{n+2}\right] \zeta_{\mathrm{S}}
$$

where $\Delta q_{0}\left(=2220 \mathrm{~m}^{2}\right.$ year $\left.^{-1}\right)$ is obtained from Equation (6) for $h=h_{0}=254 \mathrm{~m}$, and $\alpha_{0}$ is the surface slope at the $S_{2}$ bore-hole site, taken as $5.7^{\circ}$. Derivation of Equation (7) from Equation (6) involves the assumption that the channel shape factor (Paterson and Savage, I 963 , p. 4 539) remains constant, which is reasonable as an approximation since the range of channel cross-sectional shapes is not great (Fig. 2). It also assumes that the longitudinal stress state, and longitudinal gradients thereof, do not appreciably affect the flow. This type of flow has been called "laminar" (Nye, I952, p. 68). Corresponding to Equation (7), the bed slip rate $u_{\mathrm{B}}$ is given by

$$
u_{\mathrm{B}}=u_{\mathrm{S}}-\Delta u_{\mathrm{o}}\left(\frac{\sin }{\sin } \frac{\alpha}{\alpha_{0}}\right)^{n}\left(\frac{h}{h_{0}}\right)^{n+\mathrm{I}}
$$

where $\Delta u_{0}=42.7 \mathrm{~m}$ year ${ }^{-1}$. $Q_{\mathrm{F}}$ and $u_{\mathrm{B}}$ values calculated from Equations (7) and (8) at Io points along the stream line are given in Table V. These are points where the surface velocity is established by measurement at a nearby marker or by reliable interpolation between adjacent profiles from the $u_{\mathrm{S}}(\xi)$ curve (Fig. 8). At some points the ice thickness $h$ is well controlled by a nearby seismic reflection, whereas at others (for which $h$ is given in parentheses in Table V), interpolation between reflections is required. The $h$ values are read from the curve $h(\xi)$ in Figure 8, which is obtained from the surface and bedrock profiles of Figure 2, shown at an enlarged vertical scale in Figure 8 , with control points indicated. The surface slopes $\alpha$ used in calculating $Q_{\mathrm{F}}$ and listed in Table $\mathrm{V}$ are averages over $200 \mathrm{~m}$ intervals $\Delta \xi$ (approximately the ice thickness), while the curve $\alpha(\xi)$ in Figure 8 is based on roo m intervals.

Significance of discrepancies between $Q_{\mathrm{F}}$ and $Q_{\mathrm{C}}$

The $Q_{\mathrm{F}}$ values depart by as much as $\mathrm{I}_{5} \%\left(\mathrm{I} .5 \times \mathrm{IO}^{3} \mathrm{~m}^{3}\right.$ year-1) from $Q_{\mathrm{C}}(\xi)$ (Table V and Fig. 9). Measurement errors are not the main source of the large discrepancies. Errors of about $0.5 \mathrm{~m}$ year-1 in $u$ and $w$ (Table $\mathrm{I}$ ) would cause errors of about $0 . \mathrm{I} \times 10^{3} \mathrm{~m}^{3}$ year $^{-1}$ in the decrement in $Q_{\mathrm{C}}$ from one profile to the next, giving an accumulated error of about $0.3 \times \mathrm{ro}^{3} \mathrm{~m}^{3}$ year-1 in $Q_{\mathrm{C}}$ at the $a$ profile. Strain-rate error causes errors in $\zeta_{\mathrm{s}}(\xi)$ amounting to $\pm 0.02 \mathrm{~m}$ per I $00 \mathrm{~m}$ integration interval, which affect $Q_{\mathrm{F}}$ directly and $Q_{\mathrm{C}}$ incrementally;

* Note, however, that Paterson and Savage (1963) and Nye (1953; 1957) find higher values of $n$, from 4 to 5 . At bore holes $K, P_{2}$, and $\mathcal{N}$, near bore hole $\mathcal{F}$ in Blue Glacier (Fig. 1), the flow law has $n=5.2$ (Kamb and Shreve, 1966). From data for five bore holes in the general area of the stream sheet between profiles $g$ and $i$, Shreve and Sharp (1970, p. 84) deduce $n=3 \cdot 3$, but this value does not differ significantly, within the scatter of the data, from the value 2.9 used in the present calculations. 
near the $a$ profile the resulting possible errors in $Q_{\mathrm{F}}( \pm 6 \%)$ and $Q_{\mathrm{C}}(\approx \pm 2 \%)$ are significant in relation to the observed discrepancies between $Q_{\mathrm{F}}$ and $Q_{\mathrm{C}}$, but the occurrence of large discrepancies near $\xi=\mathrm{o}$ ( $\mathrm{g}$ and $i$ profiles), where the errors in $\zeta_{\mathrm{s}}$ must be small, shows that $\zeta_{\mathrm{s}}$ error is not the main source of the discrepancies. An error in ice thickness $\Delta h=10 \mathrm{~m}$ will given an error in $Q_{\mathrm{F}}$ of approximately $u_{\mathrm{B}} \Delta h$, which amounts to $0 . \mathrm{I} \times \mathrm{IO}^{3} \mathrm{~m}^{3}$ year $^{-1}$ or less for profiles $c-f$ and $i$, much less than the discrepancies between $Q_{\mathrm{F}}$ and $Q_{\mathrm{C}}$ there. Larger errors in $h$ are possible at points where the thickness is interpolated between seismic soundings, but large discrepancies between $Q_{\mathrm{F}}$ and $Q_{\mathrm{C}}$ occur both for points with well-controlled and with interpolated $h$.
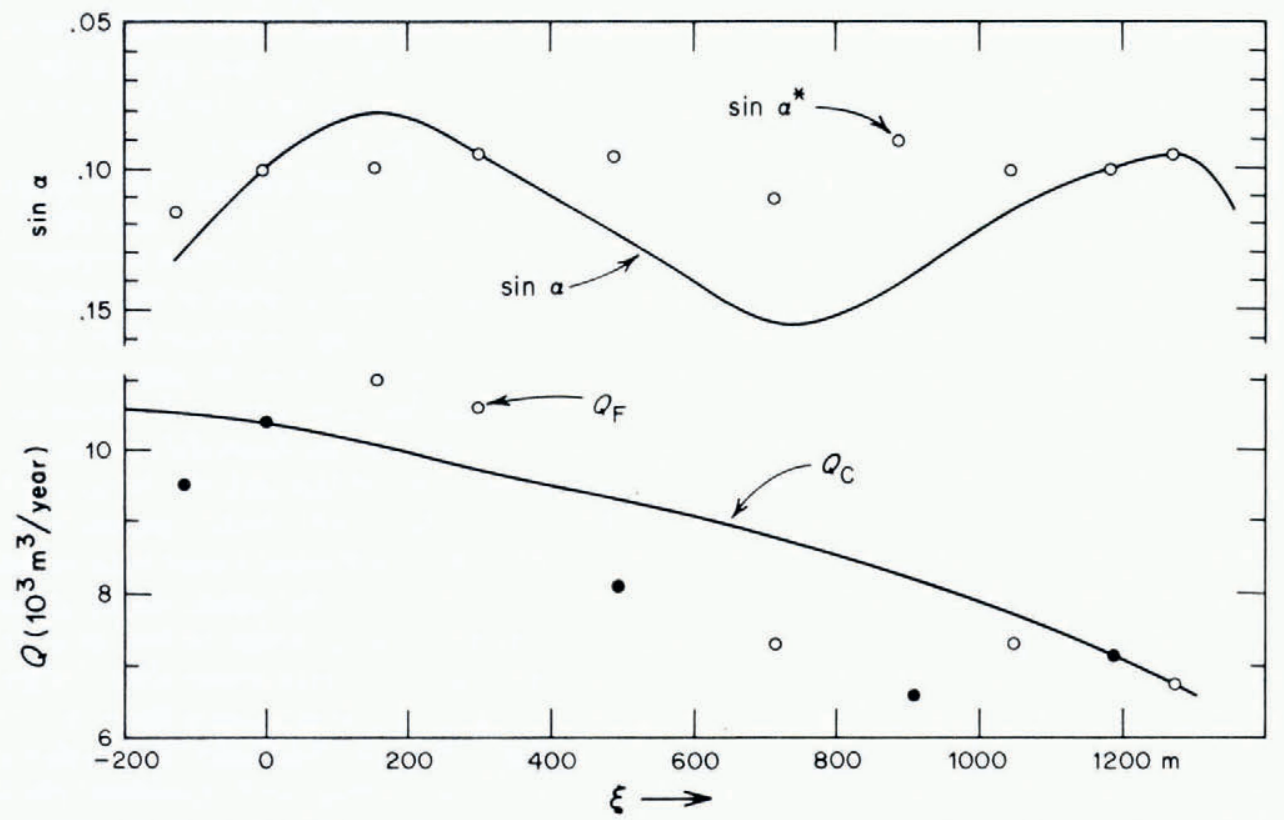

Fig. 9. Results of ice flux calculations: flux in the stream sheet is shown as a function of longitudinal coordinate $\xi$. Q $Q_{\mathrm{C}}$ (continuous curve) is calculated from continuity, and $Q_{\mathrm{F}}$ (points) from surface flow velocity and the assumed flow law. Solid circles represent points where ice thickness $h$ is well determined, open circles where $h$ is interpolated between determinations. Upper curve shows $\sin \alpha(200 \mathrm{~m}$ average $)$ as a function of $\xi$, plotted with increasing slope downward; the associated open circles show the effective slope values $\alpha^{\star}$ (see text).

Since the reach studied lies below the firn line, the erroneous down-stream increase in $Q_{F}$ of $\mathrm{I} .5 \times 1 \mathrm{IO}^{3} \mathrm{~m}^{3}$ year $^{-\mathrm{I}}$ from $\xi=-\mathrm{II} 5$ to $+\mathrm{I} 60 \mathrm{~m}$ and of $0.7 \times 10^{3} \mathrm{~m}^{3}$ year $^{-1}$ from $\xi=900$ to I $050 \mathrm{~m}$ shows that the $Q_{\mathrm{F}}$ calculation contains errors comparable to the discrepancies between $Q_{\mathrm{F}}$ and $Q_{\mathrm{C}}$. The source of the discrepancies is thus mainly in the $Q_{\mathrm{F}}$ calculation. This calculation would be invalidated if deformations parallel to the surface caused the velocity profile $u(z)$ to depart significantly from the assumed form of Equation (6), but the observed discrepancies between $Q_{\mathrm{F}}$ and $Q_{\mathrm{C}}$ are opposite to what could be expected on this account: the relatively high surface strain-rates near $c$ and $d\left(\epsilon=\left(2 \sum \dot{e}_{i j^{2}}\right)^{\frac{1}{2}}=0\right.$. I 2 year-1 at $(x, y)=(650 \mathrm{~m}$, I $650 \mathrm{~m}))$ should result in internal deformations greater than calculated on the basis of $u(z)$ at $S_{2}$ (where $\epsilon=0.05$ year $^{-1}$ ), whereas the actual internal deformations must be less than calculated because $Q_{\mathrm{F}}<Q_{\mathrm{C}}$ at $c$ and $d$; conversely, $Q_{\mathrm{F}}>Q_{\mathrm{C}}$ at $f$ and $g$, where the surface strain-rates are low $\left(\epsilon=\right.$ o.o I year $\left.{ }^{-1}\right)$. Failure of the assumption as to the shape of the stream sheet at depth could lead to errors in the comparison of $Q_{\mathrm{C}}$ and $Q_{\mathrm{F}}$ that 
are difficult to assess, but which doubtless would vary only slowly and smoothly with $\xi$, in contrast to the pattern of abrupt variation shown by $Q_{F}$ in Figure 9.

The main source of trouble in the $Q_{\mathrm{F}}$ calculation is suggested by the similarity shown in Figure 9 between the curve $\alpha(\xi)$ and the pattern of deviation of the $Q_{\mathrm{F}}$ values from $Q_{\mathrm{C}}(\xi)$. Where the surface slope $\alpha$ is small (profiles $f$ and $g$ ), the calculated fluxes $Q_{\mathrm{F}}$ are high. Where $\alpha$ is large, $Q_{\mathrm{F}}$ is low, and the calculated basal sliding velocities $u_{\mathrm{B}}$ are negative, which is inadmissible (Table V, profiles $c, d, e$, and $i$ ). A smaller ice thickness could rectify the negative $u_{\mathrm{B}}$ values, but since $Q_{\mathrm{F}}$ at these profiles is near its maximum as a function of $h$ (which occurs where $u_{\mathrm{B}}=\mathrm{o}$ ), no choice of $h$ is able to increase $Q_{\mathrm{F}}$ enough to even approach agreement with the substantially larger $Q_{\mathrm{C}}$ values there.

The primary trouble is evidently an exaggerated dependence of $Q_{\mathrm{F}}$ and $u_{\mathrm{B}}$ on $\alpha$ in Equations (7) and (8). The extent of exaggeration is shown by finding the effective slope $\alpha^{\star}$ such that, when used in place of $\alpha$ in Equation (7), agreement between $Q_{\mathrm{F}}$ and $Q_{\mathrm{C}}$ is achieved. The effective slopes $\alpha^{\star}$ correspond via Equation (8) to basal sliding rates $u_{\mathrm{B}}{ }^{\star}$ that are more reasonable than the rates $u_{\mathrm{B}}$ based on the observed surface slope $\alpha$ (Table V).

The $\alpha^{\star}$ values show substantially less variation than $\alpha$ (Table V and Fig. 9). They indicate that the internal deformation of the glacier over the reach studied is better described by treating the surface slope as constant than by taking into account its actual longitudinal variation. Since the internal deformation reflects the shear stresses parallel to the surface at depth, most importantly near the bottom, it follows that these stresses respond little if at all to local variations in surface slope. This conclusion is somewhat similar to the one reached by Budd (1968; see Nye, 1969, p. 212 ), that in large ice sheets the basal shear stress is determined by a longitudinal average of the surface slope over distances of order 20 times the ice thickness. An average over $20 h(4 \mathrm{~km})$ in Blue Glacier, which would extend through the steep ice fall and into the accumulation basins above, is not reasonable. Moreover, near constancy of $\alpha^{\star}$ does not imply near constancy of basal shear stress, because ice thickness varies substantially $\left(174^{-255} \mathrm{~m}\right)$ over the reach studied. The mechanical situation in this small valley glacier, where $\alpha$ and $h$ vary greatly over distances of order $20 h$, is not closely similar to that in a large ice sheet. Nevertheless there seems to be validity in the qualitative conclusion that the shear stresses and deformation at depth are mainly governed not by the local surface slope but by a longitudinal average over distances much larger than the ice thickness. Longitudinal averaging is accomplished mechanically by the action of longitudinal stress gradients (Budd, I970), whose importance is thus emphasized by the present results. Extending the longitudinal averaging interval from $200 \mathrm{~m}$ (as used for $\alpha$ in Table V and Figure 9) to $700 \mathrm{~m}$ does not produce detailed agreement between $\alpha$ and $\alpha^{\star}$. Better agreement, but still not complete, is obtained by equating $h \sin \alpha^{\star}$ to a longitudinal average of $h \sin \alpha$, which would be expected mechanically. It appears that if $\alpha^{\star}$ is indeed a longitudinal average, the averaging process is not everywhere simple and symmetrical about the point to which it applies.

The $\alpha^{\star}$ value for the $i$ profile is intermediate between the local $\alpha$ and the roughly constant value $\alpha^{\star} \approx 0$. Io applicable to the flow down-stream (Fig. 9). It appears that the internal deformation at $i$ already responds partially to the generally increased surface slopes upstream $(\sin \alpha \approx 0.2$ for $900 \mathrm{~m}$ up-stream, with even higher slopes above).

\section{FACtors Controlling Flow VElocity}

Although the local surface slope seems to have little effect on ice deformation versus depth (Section 8), nevertheless it does have a definite influence on the surface flow velocity, as shown by the strain-rate field and pattern of crevassing near the $c$ and $d$ profiles (Section 7 ). Influence of ice thickness is suggested by the decrease in velocity down-stream from the $c$ profile, where ice thinning toward the terminus becomes pronounced. The plot of measured center-line velocities versus $\sin \alpha$ and $h$ in Figure ro shows that the velocities behave in a systematic way 
in relation to these variables, such that contours of $u(\sin \alpha, h)$ can be drawn. Velocities from the $i$ to $d$ profiles (represented by the numbers 49-52 in Figure ro) show little longitudinal variation and fall near the $50 \mathrm{~m} /$ year contour, suggesting that over this reach the variations in $\alpha$ and $h$ have compensating effects on $u$. Velocities for the $c$ to $a$ profiles (44-33 in Figure ro) plot in succession away from the $50 \mathrm{~m} /$ year contour, suggesting that both $h$ and $\alpha$ contribute to the velocity decrease in this reach; the suggested contribution is non-linear in a curious way.

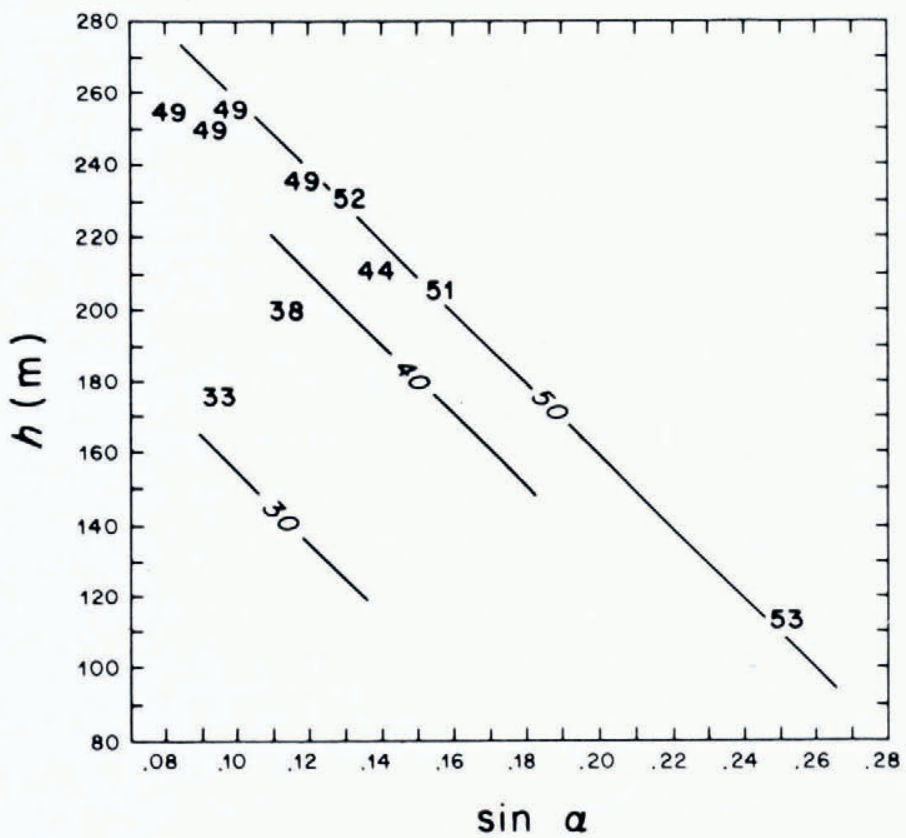

Fig. ro. Center-line surface velocities u plotted against center-line ice thickness $h$ and surface slope $\propto$ (20o m average). Measured velocities are heavy numbers; the velocity $53 \mathrm{~m} /$ year at lower right was measured at borehole $P_{2}\left(F_{i g} .1\right)$ in $I_{96} 6_{4}-65$. Contours are drawn to conform approximately to the velocity data.

The seeming implication in Figure so that $u$ is a function only of the local variables $h$ and $\alpha$, as has often been assumed in glacier-flow theory, must be viewed with suspicion, since it contradicts the conclusion that longitudinal stress gradients influence the flow (Section 8), and since it overlooks the possible effects of other variables on the sliding contribution. These additional influences would appear in failure of data points from other glaciers to conform to the simple pattern in Figure Io.

If the flow contribution from internal deformation is controlled by an effective surface slope $\alpha^{\star}$ that is made constant by longitudinal stress gradients (Section 8), a mechanical explanation is needed for how the stress distribution required to control the flow in this way is achieved. In fact a strictly constant $\alpha^{\star}$ is not established, as noted for profile $i$ in Section 8, nor is the basal shear stress constant. The flow contribution from the implied basal sliding $\left(u_{\mathrm{B}}{ }^{\star}\right.$ in Table $\left.\mathrm{V}\right)$ necessitates an unconventional control mechanism, since $u_{\mathrm{B}}{ }^{\star}$ generally decreases (though erratically) with increasing $h$ and therefore with increasing basal shear stress. The effects of longitudinally varying bedrock roughness and "lubrication" could be responsible for such behavior. We conclude that the flow is controlled by many factors in a complex way that is poorly understood, even though it shows a simple, systematic pattern in relation to ice thickness and surface slope (Fig. Io).

$M S$. received 6 June $197^{2}$ 


\section{REFERENCES}

Allen, C. R., and others. 1960. Structure of the lower Blue Glacier, Washington, by C. R. Allen, W. B. Kamb, M. F. Meier and R. P. Sharp. Journal of Geology, Vol. 68, No. 6, p. 601-25.

Budd, W. F. I968. The longitudinal velocity profile of large ice masses. Union de Géodésie et Géophysique Internationale. Association Internationale d'Hydrologie Scientifique. Assemblée générale de Berne, 25 sept.-7 oct. 1967. [Commission de Neiges et Glaces.] Rapports et discussions, p. 58-77.

Budd, W. F. 1970. The longitudinal stress and strain-rate gradients in ice masses. Journal of Glaciology, Vol. 9, No. 55 , p. $19^{-27}$.

Corbató, C. E. 1965. Thickness and basal configuration of lower Blue Glacier, Washington, determined by gravimetry. Fournal of Glaciology, Vol. 5 , No. 41, p. 637-50.

Glen, J. W. 1955. The creep of polycrystalline ice. Proceedings of the Royal Society, Ser. A, Vol. 228, No. I 175 , p. $5^{1} 9^{-38}$.

Glen, J. W., and Lewis, W. V. I961. Measurements of side-slip at Austerdalsbreen, 1959. Journal of Glaciology, Vol. 3 , No. 30, p. 1 109-22.

Harrison, W. D. 1972. Temperature of a temperate glacier. Fournal of Glaciology, Vol. I1, No. 61, p. 15-29.

Kamb, W. B., and Meier, M. F. Unpublished. Flow mechanism counteracting ablation in valley glaciers.

Kamb, W. B., and Shreve, R. L. I 966 . Results of a new method for measuring internal deformation in glaciers. Transactions. American Geophysical Union, Vol. 47, No. 1, p. 190. [Abstract.]

LaChapelle, E. R. 1959. Annual mass and energy exchange on the Blue Glacier. Journal of Geophysical Research, Vol. 64 , No. 4, p. $443-49$.

LaChapelle, E. R. I960. The Blue Glacier Project 1959 and 196o. Seattle, University of Washington. Department of Meteorology and Climatology. (Final Report, U.S. Office of Naval Research Contract 477 (18) (NR307-244).)

Mathews, W. H. r959. Vertical distribution of velocity in Salmon Glacier, British Columbia. Journal of Glaciology, Vol. 3, No. 26, p. $44^{8-54}$.

Meier, M. F. 1960. Mode of flow of Saskatchewan Glacier, Alberta, Canada. U.S. Geological Survey. Professional Paper 35 I.

Meier, M. F., and Tangborn, W. V. 1965. Net budget and flow of South Cascade Glacier, Washington. Journal of Glaciology, Vol. 5, No. 4I, p. 547-66.

Nielsen, L. E. 1955. Regimen and flow of ice in equilibrium glaciers. Bulletin of the Geological Society of America, Vol. 66, No. I, p. I-8.

Nye, J. F. 1952. The mechanics of glacier flow. Journal of Glaciology, Vol. 2, No. 12, p. 82-93.

Nye, J. F. 1953. The flow law of ice from measurements in glacier tunnels, laboratory experiments, and the Jungfraufirn borehole experiment. Proceedings of the Royal Society, Ser. A, Vol. 219, No. I I39, p. 477-89.

Nye, J. F. 1957. The distribution of stress and velocity in glaciers and ice-sheets. Proceedings of the Royal Society, Ser. A, Vol. 239, No. 1216 , p. $113-33$.

Nye, J. F. 1969. The effect of longitudinal stress on the shear stress at the base of an ice sheet. Fournal of Glaciology, Vol. 8, No. 53, p. 207-13.

Paterson, W. S. B., and Savage, J. C. 1963. Measurements on Athabasca Glacier relating to the flow law of ice. Journal of Geophysical Research, Vol. 68, No. 15, p. 4537-43.

Raymond, C. F. r97I. Flow in a transverse section of Athabasca Glacier, Alberta, Canada. Fournal of Glaciology, Vol. IO, No. $5^{8,}$, p. $55^{-84}$.

Savage, J. C., and Paterson, W. S. B. 1963. Borehole measurements in the Athabasca Glacier. Fournal of Geophysical Research, Vol. 68 , No. ${ }^{1} 5$, p. $4521-36$.

Schimpp, O. 1958. Der Eishaushalt am Hintereisferner in den Jahren 1952-53 und 1953-54. Union Géodésique et Géophysique Internationale. Association Internationale d'Hydrologie Scientifique. Assemblée générale de Toronto, 3-14 sept. 1957. Tom. 4, p. 301-14.

Shreve, R. L., and Sharp, R. P. 1970. Internal deformation and thermal anomalies in lower Blue Glacier, Mount Olympus, Washington, U.S.A. Journal of Glaciology, Vol. 9, No. 55, p. 65-86. 